\title{
Alkenylzirconocene-Mediated Preparation of
}

\author{
Alkenylphosphines
}

Taichi Miyaji, Zhenfeng Xi, Masamichi Ogasawara, Kiyohiko Nakajima, and Tamotsu Takahashi*

Catalysis Research Center and Graduate School of Pharmaceutical Sciences, Hokkaido University, and SORST, Japan Science and Technology Agency (JST), Kita-ku, Sapporo 001-0021, Japan, Department of Chemistry, Peking University, Beijing 100871, China, and Department of Chemistry, Aichi University of Education, Igaya, Kariya, Aichi, 448-8542, Japan

Supporting Informations

\section{Table of Contents}

General Section. . . . . . . . . . . . . . . . . . . . S2

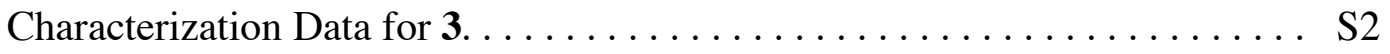

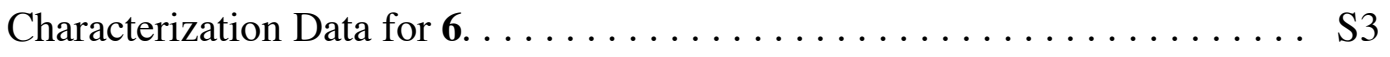

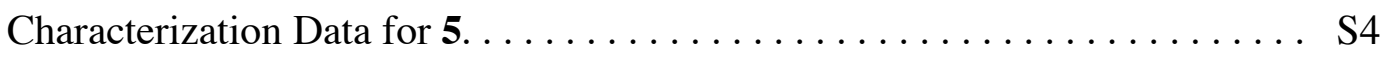

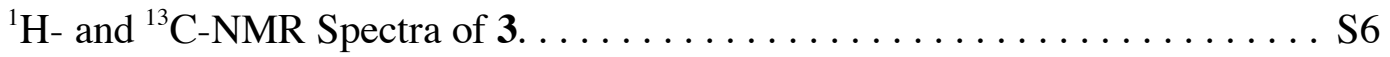

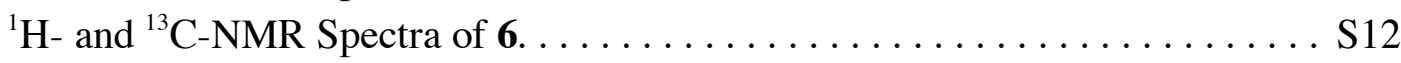

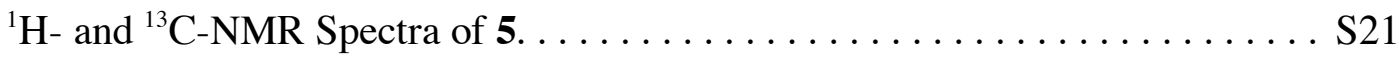


General. All anaerobic and/or moisture sensitive manipulations were carried out with standard Schlenk techniques under predried nitrogen. NMR spectra were recorded on a JEOL JNM AL300 spectrometer $\left({ }^{1} \mathrm{H}, 300 \mathrm{MHz} ;{ }^{13} \mathrm{C}, 75 \mathrm{MHz}\right)$ or a Bruker ARX-400 spectrometer $\left({ }^{1} \mathrm{H}, 400 \mathrm{MHz} ;{ }^{13} \mathrm{C}, 100\right.$ $\mathrm{MHz}$ ). ${ }^{1} \mathrm{H}$ and ${ }^{13} \mathrm{C}$ chemical shifts are reported in ppm downfield of internal tetramethylsilane. Tetrahydrofuran, $\mathrm{Et}_{2} \mathrm{O}$, and hexane (homogenized with tetraglyme) were distilled from benzophenoneketyl under nitrogen prior to use. Water was degassed by three freeze-pump-thaw cycles. All the chemicals were obtained from commercial sources and used without further purification.

Diphenyl(2-phenylethenyl)phosphine (3am). Pale yellow oil. ${ }^{1} \mathrm{H}$ NMR $\left(\mathrm{CDCl}_{3}\right): \delta 6.85(\mathrm{~d}, J=10.8$ $\mathrm{Hz}, 2 \mathrm{H}), 7.12-7.48(\mathrm{~m}, 15 \mathrm{H}) .{ }^{13} \mathrm{C} \mathrm{NMR}\left(\mathrm{CDCl}_{3}\right): \delta 126.7(\mathrm{~d}), 126.8,128.5-128.7(\mathrm{~m}), 133.1(\mathrm{~d}), 137.0$ (d), 138.1 (d), 143.8 (d). ${ }^{31} \mathrm{P}$ NMR $\left(\mathrm{CDCl}_{3}\right): \delta$-12.4. IR (Neat, $\left.\mathrm{cm}^{-1}\right): 1599(\mathrm{~m}), 1585(\mathrm{~m}), 1572(\mathrm{w})$, 1479 (s), 1433 (s), 972 (s), 738 (s), 694 (s). HRMS: m/z Calcd for $\mathrm{C}_{20} \mathrm{H}_{17} \mathrm{P}: 288.1068$, Found: 288.1076.

(1-Octenyl)diphenylphosphine (3bm). Colorless oil. ${ }^{1} \mathrm{H} \mathrm{NMR}\left(\mathrm{CDCl}_{3}\right): \delta 0.88(\mathrm{t}, J=6.96 \mathrm{~Hz}, 3 \mathrm{H})$, 1.20-1.51 (m, 8H), 2.14-2.28 (m, 2H), 6.15-6.35 (m, 2H), 7.21-7.44 (m, 10H). ${ }^{13} \mathrm{C} \mathrm{NMR}\left(\mathrm{CDCl}_{3}\right): \delta 14.1$, 22.6, 28.5, 28.8, 31.6, 35.0 (d), 126.8 (d), 128.2 (d), 128.3, 132.6 (d), 139.2 (d), 149.2 (d). ${ }^{31} \mathrm{P}$ NMR $\left(\mathrm{CDCl}_{3}\right): \delta$-14.0. IR (Neat, cm $\left.{ }^{-1}\right): 1585$ (m), $1433(\mathrm{~s}), 1201(\mathrm{~m}), 972(\mathrm{~m}), 738$ (s), 696 (s). Anal. Calcd for $\mathrm{C}_{20} \mathrm{H}_{25} \mathrm{P}: \mathrm{C}, 81.05 ; \mathrm{H}, 8.50$. Found: $\mathrm{C}, 81.30 ; \mathrm{H}, 8.72$.

[Diisopropyl(2-phenylethenyl)phosphine] $\left(\mathbf{B H}_{3}\right)\left(\mathbf{3 a n}-\mathbf{B H}_{3}\right)$. A colorless solid. mp: 133-134 ${ }^{\circ} \mathrm{C} .{ }^{1} \mathrm{H}$ $\operatorname{NMR}\left(\mathrm{CDCl}_{3}\right): \delta 1.08-1.30(\mathrm{~m}, 12 \mathrm{H}), 2.00-2.21(\mathrm{~m}, 2 \mathrm{H}), 6.28(\mathrm{dd}, J=5.0$ and $17.1 \mathrm{~Hz}, 1 \mathrm{H}), 7.30-7.57$ $(\mathrm{m}, 6 \mathrm{H}) .{ }^{13} \mathrm{C} \mathrm{NMR}\left(\mathrm{CDCl}_{3}\right): \delta 16.7$ (d), 22.1 (d), 112.7 (d), 127.3, 128.8, 129.7, $135.7(\mathrm{~d}), 150.4(\mathrm{~d}) .{ }^{31} \mathrm{P}$ NMR $\left(\mathrm{CDCl}_{3}\right): \delta$ 30.8. IR (KBr, cm $\left.{ }^{-1}\right): 2393(\mathrm{~m}), 1606(\mathrm{w}), 1593$ (m), 1572 (w), 1493 (s), 1444 (s), 1074 (s), 991 (s), 740 (s), 690 (s). Anal. Calcd for $\mathrm{C}_{14} \mathrm{H}_{24} \mathrm{BP}$ : C, 71.82; H, 10.33. Found: C, 71.60; $\mathrm{H}, 10.19$.

[2-(3-Aminophenyl)ethenyl]diisopropylphosphine (3cn). Yellow viscous oil. ${ }^{1} \mathrm{H} \mathrm{NMR}\left(\mathrm{CDCl}_{3}\right): \delta$ $1.01-1.13(\mathrm{~m}, 12 \mathrm{H}), 1.84(\mathrm{dd}, J=1.8$ and $7.0 \mathrm{~Hz}, 2 \mathrm{H}), 3.66(\mathrm{br}, 2 \mathrm{H}), 6.50(\mathrm{dd}, J=2.9$ and $17.3 \mathrm{~Hz}, 1 \mathrm{H})$, $6.58(\mathrm{ddd}, J=0.9,2.4$, and $7.9 \mathrm{~Hz}, 1 \mathrm{H}), 6.74(\mathrm{dd}, J=1.8$ and $2.4 \mathrm{~Hz}, 1 \mathrm{H}), 6.84(\mathrm{~d}, J=7.7 \mathrm{~Hz}, 1 \mathrm{H}), 6.96$ $(\mathrm{dd}, J=13.6$ and $17.3 \mathrm{~Hz}, 1 \mathrm{H}), 7.11(\mathrm{dd}, J=7.7$ and $7.9 \mathrm{~Hz}, 1 \mathrm{H}) .{ }^{13} \mathrm{C} \mathrm{NMR}\left(\mathrm{CDCl}_{3}\right): \delta 18.7(\mathrm{~d}), 19.9$ (d), 23.6 (d), 113.0, 115.1, 117.3, 125.2 (d), 129.5, 138.4 (d), 145.8 (d), 146.7. ${ }^{31} \mathrm{P}$ NMR $\left(\mathrm{CDCl}_{3}\right): \delta 4.7$. IR (Neat, cm ${ }^{-1}$ ): 3452 (m), 3360 (s), 3129 (m), 1620 (s), 1581 (s), 1495 (s), 1458 (s), 1304 (s), 993 (s), 773 (s). HRMS: $m / z$ Calcd for $\mathrm{C}_{14} \mathrm{H}_{22} \mathrm{NP}: 235.1490$, Found: 235.1414.

Bis(2-phenylethenyl)phenylphosphine (3ao). A pale yellow solid. mp: 82-83 ${ }^{\circ} \mathrm{C} .{ }^{1} \mathrm{H} \mathrm{NMR}\left(\mathrm{CDCl}_{3}\right)$ : $\delta 6.82(\mathrm{dd}, J=7.9$ and $17.3 \mathrm{~Hz}, 2 \mathrm{H}), 7.02(\mathrm{dd}, J=13.8$ and $17.3 \mathrm{~Hz}, 2 \mathrm{H}), 7.34-7.58(\mathrm{~m}, 15 \mathrm{H}) .{ }^{13} \mathrm{C}$ NMR $\left(\mathrm{CDCl}_{3}\right): \delta$ 126.80, 126.81 (d), 128.4, 128.55, 128.58, 128.7, 137.0 (d), 138.3 (d), 132.3 (d), 143.0 (d). ${ }^{31} \mathrm{P}$ NMR $\left(\mathrm{CDCl}_{3}\right): \delta$ 18.1. IR (Neat, $\left.\mathrm{cm}^{-1}\right): 1606$ (s), 1597 (s), 1574 (s), 1493 (s), 1444 (s), 989 (s), 979 (s), 966 (s), 796 (m), 746 (s), 698 (s). HRMS: m/z Calcd for $\mathrm{C}_{22} \mathrm{H}_{19} \mathrm{P}: 314.1224$, Found: 314.1209.

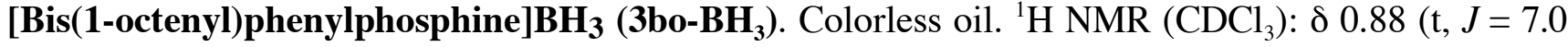
$\mathrm{Hz}, 6 \mathrm{H}), 1.15-1.57(\mathrm{~m}, 16 \mathrm{H}), 2.16-2.35(\mathrm{~m}, 4 \mathrm{H}), 5.80-5.98(\mathrm{~m}, 2 \mathrm{H}), 6.45-6.68(\mathrm{~m}, 2 \mathrm{H}), 7.33-7.52(\mathrm{~m}$, $3 \mathrm{H}), 7.56-7.73(\mathrm{~m}, 2 \mathrm{H}) .{ }^{13} \mathrm{C} \mathrm{NMR}\left(\mathrm{CDCl}_{3}\right): \delta 14.0,22.6,28.0,28.8,31.6,34.8(\mathrm{~d}), 118.7$ (d), 128.7 (d), 130.72 (d), 130.74 (d), 131.7 (d), 151.9 (d). ${ }^{31} \mathrm{P}$ NMR $\left(\mathrm{CDCl}_{3}\right): \delta$ 5.8. IR (Neat, $\left.\mathrm{cm}^{-1}\right): 2382$ (s), 1627 
(m), $1620(\mathrm{~m}), 1575(\mathrm{~m}), 1476$ (s), 1109 (m), 1059 (s), 974 (m), 742 (s), 694 (s). Anal. Calcd for $\mathrm{C}_{22} \mathrm{H}_{38}$ BP: C, 76.74; H, 11.12. Found: C, 76.54; H, 11.23.

Diphenyl(propen-2-yl)phosphine (6am). Colorless oil. ${ }^{1} \mathrm{H} \mathrm{NMR}\left(\mathrm{CDCl}_{3}\right): \delta 1.86(\mathrm{ddd}, J=1.1,1.7$, and $9.0 \mathrm{~Hz}, 3 \mathrm{H}$ ), 5.08 (qdd, $J=1.1,1.7$, and $11.0 \mathrm{~Hz}, 1 \mathrm{H}$ ), 5.61 (qdd, $J=1.6,1.7$, and $15.0 \mathrm{~Hz}, 1 \mathrm{H}$ ), 7.21-7.43 (m, 10H). ${ }^{13} \mathrm{C} \mathrm{NMR}\left(\mathrm{CDCl}_{3}\right): \delta 22.3(\mathrm{~d}), 125.0(\mathrm{~d}), 128.4(\mathrm{~d}), 128.7,133.7$ (d), $135.8(\mathrm{~d})$, 143.6 (d). ${ }^{31} \mathrm{P}$ NMR $\left(\mathrm{CDCl}_{3}\right): \delta$-1.0. IR (Neat, $\left.\mathrm{cm}^{-1}\right): 1599(\mathrm{w}), 1587(\mathrm{~m}), 1435$ (s), $1261(\mathrm{~m}), 742$ (s), 696 (s). HRMS: $m / z$ Calcd for $\mathrm{C}_{15} \mathrm{H}_{15} \mathrm{P}: 226.0911$, Found: 226.0915.

(4-Octen-4-yl)diphenylphosphine (6bm). Colorless oil. ${ }^{1} \mathrm{H} \mathrm{NMR}\left(\mathrm{CDCl}_{3}\right): \delta 0.84(\mathrm{t}, J=7.3 \mathrm{~Hz}, 3 \mathrm{H})$, $0.95(\mathrm{t}, J=7.3 \mathrm{~Hz}, 3 \mathrm{H}), 1.27-1.49(\mathrm{~m}, 4 \mathrm{H}), 2.16-2.25(\mathrm{~m}, 4 \mathrm{H}), 5.59(\mathrm{td}, J=7.2$ and $12.8 \mathrm{~Hz}, 1 \mathrm{H}), 7.34-$ $7.45(\mathrm{~m}, 10 \mathrm{H}) .{ }^{13} \mathrm{C}$ NMR $\left(\mathrm{CDCl}_{3}\right): \delta 13.8,14.2,22.6,23.0(\mathrm{~d}), 31.2(\mathrm{~d}), 33.3(\mathrm{~d}), 128.2(\mathrm{~d}), 128.3,133.7$ (d), 136.9 (d), 137.1 (d), 141.5 (d). ${ }^{31} \mathrm{P}$ NMR $\left(\mathrm{CDCl}_{3}\right): \delta 0.7 . \mathrm{IR}\left(\mathrm{KBr}, \mathrm{cm}^{-1}\right): 1585$ (m), 1479 (m), 1433 (s), 1091 (m), 908 (m), 742 (s), 696 (s). HRMS: m/z Calcd for $\mathrm{C}_{20} \mathrm{H}_{25} \mathrm{P}: 296.1694$, Found: 296.1681.

(4-Ethyl-3-hexen-3-yl)diphenylphosphine (6cm). Colorless oil. ${ }^{1} \mathrm{H}$ NMR $\left(\mathrm{CDCl}_{3}\right): \delta 0.34(\mathrm{t}, J=7.5$ $\mathrm{Hz}, 3 \mathrm{H}), 1.05(\mathrm{t}, J=7.5 \mathrm{~Hz}, 3 \mathrm{H}), 1.11(\mathrm{t}, J=7.5 \mathrm{~Hz}, 3 \mathrm{H}), 2.10-2.19(\mathrm{~m}, 2 \mathrm{H}), 2.27(\mathrm{q}, J=7.5 \mathrm{~Hz}, 2 \mathrm{H})$, $2.62(\mathrm{q}, J=7.5 \mathrm{~Hz}, 2 \mathrm{H}), 7.28-7.40(\mathrm{~m}, 10 \mathrm{H}) .{ }^{13} \mathrm{C} \mathrm{NMR}\left(\mathrm{CDCl}_{3}\right): \delta 13.5,14.0,14.7,24.2(\mathrm{~d}), 25.3(\mathrm{~d})$, 26.8 (d), 127.8, 128.1 (d), 128.8 (d), 133.2 (d), 138.0 (d), 158.1 (d). ${ }^{31} \mathrm{P}$ NMR $\left(\mathrm{CDCl}_{3}\right)$ : $\delta$-10.9. IR (Neat, $\mathrm{cm}^{-1}$ ): 1599 (m), 1585 (m), 1477 (s), 1433 (s), 742 (s), 698 (s). Anal. Calcd for $\mathrm{C}_{20} \mathrm{H}_{25} \mathrm{P}: \mathrm{C}, 81.05$; H, 8.50. Found: C, 80.74; H, 8.52.

(4-Ethyl-3-hexen-3-yl)diisopropylphosphine (6cn). Colorless oil. ${ }^{1} \mathrm{H}$ NMR $\left(\mathrm{CDCl}_{3}\right)$ : $\delta$ 0.91-1.04 (m, $15 \mathrm{H}), 1.14(\mathrm{dd}, J=7.0$ and $14.5 \mathrm{~Hz}, 6 \mathrm{H}), 1.87$ (qqd, $J=6.8,7.0$, and 7.0 Hz, 2H), 2.14-2.23 (m, 4H), $2.58(\mathrm{dq}, J=2.6$ and $7.5 \mathrm{~Hz}, 2 \mathrm{H}) .{ }^{13} \mathrm{C} \mathrm{NMR}\left(\mathrm{CDCl}_{3}\right): \delta 13.2,14.1(\mathrm{~d}), 16.0,21.2(\mathrm{~d}), 21.4(\mathrm{~d}), 23.2(\mathrm{~d})$, 23.8 (d), 25.0 (d), 26.2 (d), 130.3 (d), 158.6 (d). ${ }^{31} \mathrm{P}$ NMR $\left(\mathrm{CDCl}_{3}\right): \delta$ 7.2. IR $\left(\mathrm{Neat}, \mathrm{cm}^{-1}\right) 1603$ (m), 1462 (s), 1381 (s), 1261 (s), 877 (m), 806 (s), 646 (s). HRMS: m/z Calcd for $\mathrm{C}_{14} \mathrm{H}_{29} \mathrm{P}:$ 228.2007, Found: 228.2005.

(5-Propyl-4,7-octadien-4-yl)diisopropylphosphine (6dn). Colorless oil. ${ }^{1} \mathrm{H} \mathrm{NMR}\left(\mathrm{CDCl}_{3}\right)$ : $\delta 0.90$ $0.96(\mathrm{~m}, 12 \mathrm{H}), 1.14(\mathrm{dd}, J=7.0$ and $14.7 \mathrm{~Hz}, 6 \mathrm{H}), 1.33-1.50(\mathrm{~m}, 4 \mathrm{H}), 1.86(\mathrm{qqd}, J=6.8,7.0$, and $7.0 \mathrm{~Hz}$, 2H), 2.08-2.15 (m, 4H), 3.39-3.43 (m, 2H), 4.94-5.04 (m, 2H), 5.74 (tdd, $J=6.6,9.9$, and 17.1 Hz, 1H). ${ }^{13} \mathrm{C} \mathrm{NMR}\left(\mathrm{CDCl}_{3}\right): \delta$ 14.5, 15.0, $21.2(\mathrm{~d}), 21.4(\mathrm{~d}), 21.6,23.8(\mathrm{~d}), 24.6,33.5$ (d), 34.3 (d), 38.0 (d), 115.1, 132.1 (d), 137.4, 153.1 (d). ${ }^{31} \mathrm{P}$ NMR $\left(\mathrm{CDCl}_{3}\right): \delta$ 6.9. IR (Neat, $\left.\mathrm{cm}^{-1}\right): 1637(\mathrm{~m}), 1597(\mathrm{~m}), 1460$ (s), 993 (s), 908 (s),877 (m), 806 (m); HRMS: $m / z$ Calcd for $\mathrm{C}_{17} \mathrm{H}_{33} \mathrm{P}: 268.2320$, Found: 268.2332.

(1,2-Diphenyl-1,4-pentadien-1-yl)diisopropylphosphine (6en). Colorless viscous oil. ${ }^{1} \mathrm{H}$ NMR $\left(\mathrm{CDCl}_{3}\right): \delta 1.05(\mathrm{dd}, J=6.8$ and $14.0 \mathrm{~Hz}, 6 \mathrm{H}), 1.08(\mathrm{dd}, J=7.0$ and $14.1 \mathrm{~Hz}, 6 \mathrm{H}), 1.78(\mathrm{qqd}, J=6.8$, 7.0, and 7.0 Hz, 2H), 3.92-3.96 (m, 2H), $4.97(\mathrm{ddd}, J=1.3,1.7$, and 10.3 Hz, 1H), 5.05 (qd, $J=1.7$ and $17.0 \mathrm{~Hz}, 1 \mathrm{H}), 5.77(\mathrm{tdd}, J=6.8,10.3$, and $17.0 \mathrm{~Hz}, 1 \mathrm{H}), 6.80-7.05(\mathrm{~m}, 10 \mathrm{H}) .{ }^{13} \mathrm{C} \mathrm{NMR}\left(\mathrm{CDCl}_{3}\right): \delta 20.5$ (d), 21.4 (d), 23.6 (d), 41.1 (d), 116.1, 125.3, 125.9, 127.2, 127.2, 128.8, 129.4, 135.8, 137.7 (d), 141.3 (d), 143.0 (d), $154.8(\mathrm{~d}) .{ }^{31} \mathrm{P}$ NMR $\left(\mathrm{CDCl}_{3}\right): \delta$ 1.2. IR (Neat, $\left.\mathrm{cm}^{-1}\right): 1639(\mathrm{w}), 1599(\mathrm{~m}), 1585(\mathrm{~m}), 1574$ (w), $1485(\mathrm{~m}), 1441$ (m), $1383(\mathrm{~m}), 910$ (m), 702 (s). Anal. Calcd for $\mathrm{C}_{23} \mathrm{H}_{29} \mathrm{P}: \mathrm{C}, 82.11 ; \mathrm{H}$, 8.69. Found C, 81.86; H, 8.94. 
(5-Cyclopropylmethyl-4-octen-4-yl)diphenylphosphine (6fm). Colorless oil. ${ }^{1} \mathrm{H}$ NMR $\left(\mathrm{CDCl}_{3}\right): \delta$ $0.18(\mathrm{dt}, J=4.8$ and $5.3 \mathrm{~Hz}, 2 \mathrm{H}), 0.39-0.46(\mathrm{~m}, 5 \mathrm{H}), 0.58-0.71(\mathrm{~m}, 2 \mathrm{H}), 0.75-0.88(\mathrm{~m}, 1 \mathrm{H}), 0.99(\mathrm{t}, J=$ $7.3 \mathrm{~Hz}, 3 \mathrm{H}), 1.50-1.63(\mathrm{~m}, 2 \mathrm{H}), 2.03-2.10(\mathrm{~m}, 2 \mathrm{H}), 2.31-2.36(\mathrm{~m}, 2 \mathrm{H}), 2.59(\mathrm{dd}, J=3.3$ and $6.6 \mathrm{~Hz}, 2 \mathrm{H})$, 7.28-7.40 (m, 10H). ${ }^{13} \mathrm{C} \mathrm{NMR}\left(\mathrm{CDCl}_{3}\right): \delta 4.8,10.9,14.3,14.7,22.2,23.5,33.9(\mathrm{~d}), 35.0$ (d), 37.9 (d), 127.9, 128.1 (d), 128.9 (d), 133.2 (d), 138.1 (d), 155.3 (d). ${ }^{31} \mathrm{P}$ NMR $\left(\mathrm{CDCl}_{3}\right): \delta$-10.6. IR $\left(\mathrm{Neat}, \mathrm{cm}^{-1}\right)$ : $1598(\mathrm{~m}), 1585$ (m), 1479 (m), 1433 (s), 1377 (m), 742 (s), 696 (s). Anal. Calcd for $\mathrm{C}_{24} \mathrm{H}_{31} \mathrm{P}: \mathrm{C}, 82.25$; H, 8.92. Found: C, 82.76; H, 9.09.

(5-Cyclopropylmethyl-4-octen-4-yl)diisopropylphosphine (6fn). Colorless oil. ${ }^{1} \mathrm{H} \mathrm{NMR}\left(\mathrm{CDCl}_{3}\right): \delta$ 0.14-0.19 (m, 2H), 0.37-0.43 (m, 2H), 0.69-0.82 (m, 1H), 0.89-0.96 (m, 12H), 1.13 (dd, $J=7.0$ and 14.5 $\mathrm{Hz}, 6 \mathrm{H}), 1.33-1.52(\mathrm{~m}, 4 \mathrm{H}), 1.85$ (qqd, $J=6.8,7.0$, and 7.0 Hz, 2H), 2.08-2.15 (m, 2H), 2.22-2.27 (m, $2 \mathrm{H}), 2.56(\mathrm{dd}, J=3.7$ and $6.8 \mathrm{~Hz}, 2 \mathrm{H}) .{ }^{13} \mathrm{C} \mathrm{NMR}\left(\mathrm{CDCl}_{3}\right): \delta 4.7,10.9,14.5,15.0,21.2(\mathrm{~d}), 21.5(\mathrm{~d})$, 21.8, 24.0 (d), 24.7, 33.5 (d), 34.7 (d), 37.4 (d), 130.7 (d), 155.9 (d). ${ }^{31} \mathrm{P} \mathrm{NMR}\left(\mathrm{CDCl}_{3}\right): \delta$ 6.3. IR (Neat, $\mathrm{cm}^{-1}$ ): 1597 (w), 1465 (s) 1456 (s), 1377 (s), 1261 (s), 1014 (s), 802 (s); HRMS: m/z Calcd for $\mathrm{C}_{18} \mathrm{H}_{35} \mathrm{P}$ : 282.2476, Found: 282.2452.

(3-Cyclomethyl-1,2-diphenylpropen-1-yl)diisopropylphosphine (6gn). Colorless viscous oil. ${ }^{1} \mathrm{H}$ NMR $\left(\mathrm{CDCl}_{3}\right): \delta$ 0.06-0.11 (m, 2H), 0.29-0.35 (m, 2H), 0.63-0.76 (m, 1H), $1.03(\mathrm{dd}, J=6.8$ and 13.8 $\mathrm{Hz}, 6 \mathrm{H}), 1.10(\mathrm{dd}, J=7.0$ and $14.3 \mathrm{~Hz}, 6 \mathrm{H}), 1.77(\mathrm{qqd}, J=6.8,7.0$, and $7.0 \mathrm{~Hz}, 2 \mathrm{H}), 3.05(\mathrm{dd}, J=3.7$ and $6.8 \mathrm{~Hz}, 2 \mathrm{H}), 6.81-7.06(\mathrm{~m}, 10 \mathrm{H}) .{ }^{13} \mathrm{C} \mathrm{NMR}\left(\mathrm{CDCl}_{3}\right): \delta 4.8,10.3,20.3(\mathrm{~d}), 21.4(\mathrm{~d}), 23.4(\mathrm{~d}), 40.7$ (d), 125.2, 125.7, 127.1, 127.2, 128.8, 129.6, 136.2 (d), 141.4 (d), 143.7 (d), 157.9 (d). ${ }^{31} \mathrm{P}$ NMR $\left(\mathrm{CDCl}_{3}\right)$ : $\delta$-1.0. IR (Neat, $\left.\mathrm{cm}^{-1}\right): 1599(\mathrm{~m}), 1584(\mathrm{~m}), 1577(\mathrm{w}), 1487(\mathrm{~s}), 1441(\mathrm{~m}), 1016(\mathrm{~m}), 765$ (s), 700 (s). HRMS: $m / z$ Calcd for $\mathrm{C}_{24} \mathrm{H}_{31} \mathrm{P}: 350.2163$, Found: 350.2173.

[(Diphenyl(propen-2-yl)phosphine) $\mathbf{C u}(\mu \text {-Cl) }]_{2}$ (5am). Colorless crystals. mp: $151-152{ }^{\circ} \mathrm{C}(\mathrm{dec}) .{ }^{1} \mathrm{H}$ $\operatorname{NMR}\left(\mathrm{CDCl}_{3}\right): \delta 1.97(\mathrm{~d}, J=12.1 \mathrm{~Hz}, 6 \mathrm{H}), 5.14(\mathrm{~d}, J=15.0 \mathrm{~Hz}, 2 \mathrm{H}), 5.66(\mathrm{~d}, J=31.2 \mathrm{~Hz}, 2 \mathrm{H}), 7.22-$ $7.37(\mathrm{~m}, 12 \mathrm{H}), 7.63-7.69(\mathrm{~m}, 8 \mathrm{H}) .{ }^{13} \mathrm{C} \mathrm{NMR}\left(\mathrm{CDCl}_{3}\right): \delta 22.1(\mathrm{~d}), 126.8(\mathrm{~d}), 128.5(\mathrm{~d}), 129.9,131.2(\mathrm{~d})$, 134.2 (d), 139.7 (d). ${ }^{31} \mathrm{P}$ NMR $\left(\mathrm{CDCl}_{3}\right): \delta$ 0.6. IR $\left(\mathrm{KBr}, \mathrm{cm}^{-1}\right): 1587$ (w), 1479 (s), 1435 (s), 1097 (s), 910 (m), 744 (s), 698 (s). Anal. Calcd for $\mathrm{C}_{15} \mathrm{H}_{15} \mathrm{ClCuP}: \mathrm{C}, 55.39 ; \mathrm{H}, 4.65 ; \mathrm{Cl}, 10.90$. Found: C, 55.69; $\mathrm{H}, 4.75 ; \mathrm{Cl}, 10.57$.

[[(4-Ethyl-3-hexen-3-yl)diphenylphosphine $\left.] \mathbf{C u}(\mu-\mathbf{C l})]_{2} \mathbf{( 5 c m}\right)$. Colorless crystals. mp: $163-164{ }^{\circ} \mathrm{C}$. ${ }^{1} \mathrm{H} \mathrm{NMR}\left(\mathrm{CDCl}_{3}\right): \delta 0.58(\mathrm{t}, J=7.5 \mathrm{~Hz}, 6 \mathrm{H}), 0.75(\mathrm{t}, J=7.5 \mathrm{~Hz}, 6 \mathrm{H}), 1.06(\mathrm{t}, J=7.6 \mathrm{~Hz}, 6 \mathrm{H}), 2.00-2.18$ $(\mathrm{m}, 4 \mathrm{H}), 2.11-2.37(\mathrm{~m}, 4 \mathrm{H}), 2.43-2.57(\mathrm{~m}, 4 \mathrm{H}), 7.18-7.34(\mathrm{~m}, 12 \mathrm{H}), 7.42-7.52(\mathrm{~m}, 8 \mathrm{H}) .{ }^{13} \mathrm{C} \mathrm{NMR}$ $\left(\mathrm{CDCl}_{3}\right): \delta 12.5,13.1,14.5,24.7,25.3,28.6$ (d), 124.6 (d), 128.2 (d), 129.2, 132.9 (d), 133.9 (d), 157.3 (d). ${ }^{31} \mathrm{P}$ NMR $\left(\mathrm{CDCl}_{3}\right): \delta$-8.9. IR $\left(\mathrm{KBr}, \mathrm{cm}^{-1}\right): 1599$ (m), 1477 (s), 1433 (s), 1373 (m), 1093 (s), 750 (s), 698 (s). Anal. Calcd for $\mathrm{C}_{40} \mathrm{H}_{50} \mathrm{Cl}_{2} \mathrm{Cu}_{2} \mathrm{P}_{2}$ : C, 60.75; H, 6.37; Cl, 8.97. Found: C, 60.97; H,6.39; Cl, 8.76.

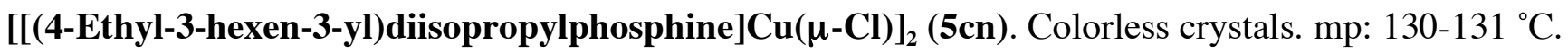
${ }^{1} \mathrm{H} \mathrm{NMR}\left(\mathrm{CDCl}_{3}\right): \delta 0.98-1.19(\mathrm{~m}, 30 \mathrm{H}), 1.34(\mathrm{dd}, J=6.6$ and $16.5 \mathrm{~Hz}, 12 \mathrm{H}), 2.05-2.32(\mathrm{~m}, 12 \mathrm{H}), 2.82$ $(\mathrm{q}, J=7.5 \mathrm{~Hz}, 4 \mathrm{H}) .{ }^{13} \mathrm{C} \mathrm{NMR}\left(\mathrm{CDCl}_{3}\right): \delta$ 13.0, 14.4, 15.5, $20.4(\mathrm{~d}), 21.0(\mathrm{~d}), 24.3,24.4(\mathrm{~d}), 25.5(\mathrm{~d})$, $27.8(\mathrm{~d}), 123.6(\mathrm{~d}), 161.3(\mathrm{~d}) .{ }^{31} \mathrm{P} \mathrm{NMR}\left(\mathrm{CDCl}_{3}\right): \delta 20.8 . \mathrm{IR}\left(\mathrm{KBr}, \mathrm{cm}^{-1}\right): 1610(\mathrm{~m}), 1460(\mathrm{~s}), 1383(\mathrm{~s})$, $1074(\mathrm{~m}), 881(\mathrm{~m}), 752(\mathrm{~m}), 688(\mathrm{~m}), 661$ (s). Anal. Calcd for $\mathrm{C}_{28} \mathrm{H}_{58} \mathrm{Cl}_{2} \mathrm{Cu}_{2} \mathrm{P}_{2}: \mathrm{C}, 51.37 ; \mathrm{H}, 8.93 ; \mathrm{Cl}$, 10.83. Found: C, 51.64; H, 8.90; Cl, 10.71 . 
[[(5-Cyclopropylmethyl-4-octen-4-yl)diphenylphospine $\left.] \mathbf{C u}(\boldsymbol{\mu}-\mathbf{C l})]_{2} \mathbf{( 5 f m}\right)$. Colorless crystals. mp: 162-163 ${ }^{\circ} \mathrm{C} .{ }^{1} \mathrm{H}$ NMR $\left(\mathrm{CDCl}_{3}\right): \delta$ 0.04-0.13 (m, 4H), 0.24-0.33 (m, 4H), $0.45(\mathrm{t}, J=7.3 \mathrm{~Hz}, 6 \mathrm{H}), 0.68-$ $0.77(\mathrm{~m}, 2 \mathrm{H}), 0.97$ (t, $J=7.4 \mathrm{~Hz}, 6 \mathrm{H}), 1.02-1.09(\mathrm{~m}, 4 \mathrm{H}), 1.48-1.59$ (m, 4H), 1.95-2.06 (m, 4H), 2.35$2.42(\mathrm{~m}, 4 \mathrm{H}), 2.49(\mathrm{~d}, J=5.2 \mathrm{~Hz}, 4 \mathrm{H}), 7.26-7.38(\mathrm{~m}, 12 \mathrm{H}), 7.55-7.67(\mathrm{~m}, 8 \mathrm{H}) .{ }^{13} \mathrm{C} \mathrm{NMR}\left(\mathrm{CDCl}_{3}\right): \delta 5.0$, 9.9, 13.9, 14.4, 22.0, 23.4, 33.4 (d), 34.6 (d), 39.6 (d), 124.0 (d), 128.3 (d), 129.6, 132.5 (d), 134.0 (d), 154.9 (d). ${ }^{31} \mathrm{P}$ NMR $\left(\mathrm{CDCl}_{3}\right): \delta$-5.7. IR $\left(\mathrm{KBr}, \mathrm{cm}^{-1}\right): 1597$ (m), 1479 (s), 1454 (s), 1437 (s), 1093 (s), 746 (s), 696 (s). Anal. Calcd for $\mathrm{C}_{48} \mathrm{H}_{62} \mathrm{Cl}_{2} \mathrm{Cu}_{2} \mathrm{P}_{2}$ : C, 64.13; H, 6.95; Cl, 7.89. Found: C, 64.45; H, 7.12; $\mathrm{Cl}, 7.45$.

[[(5-Cyclopropylmethyl-4-octen-4-yl)diisopropylphosphine $] \mathbf{C u}(\boldsymbol{\mu}-\mathbf{C l})]_{2}(\mathbf{5 f n})$. Colorless crystals. mp: 73-74 ${ }^{\circ} \mathrm{C} .{ }^{1} \mathrm{H} \mathrm{NMR}\left(\mathrm{CDCl}_{3}\right): \delta 0.31-0.35(\mathrm{~m}, 4 \mathrm{H}), 0.46-0.54(\mathrm{~m}, 4 \mathrm{H}), 0.76-0.86(\mathrm{~m}, 2 \mathrm{H}), 0.95(\mathrm{t}, J=$ $7.3 \mathrm{~Hz}, 6 \mathrm{H}), 0.97(\mathrm{t}, J=7.1 \mathrm{~Hz}, 6 \mathrm{H}), 1.08(\mathrm{dd}, J=7.0$ and $16.2 \mathrm{~Hz}, 12 \mathrm{H}), 1.32(\mathrm{dd}, J=6.8$ and $17.6 \mathrm{~Hz}$, $12 \mathrm{H}), 1.38-1.54(\mathrm{~m}, 8 \mathrm{H}), 2.06-2.21(\mathrm{~m}, 8 \mathrm{H}), 2.28-2.35(\mathrm{~m}, 4 \mathrm{H}), 2.76(\mathrm{~d}, J=5.5 \mathrm{~Hz}, 4 \mathrm{H}) .{ }^{13} \mathrm{C}$ NMR $\left(\mathrm{CDCl}_{3}\right): \delta$ 5.1, 10.9, 14.9, 15.2, 20.6 (d), $21.2(\mathrm{~d}), 21.7,24.3,24.7$ (d), 34.8, 35.1 (d), 39.4 (d), $123.5(\mathrm{~d})$, $158.7(\mathrm{~d}) .{ }^{31} \mathrm{P}$ NMR $\left(\mathrm{CDCl}_{3}\right): \delta 22.9 . \mathrm{IR}\left(\mathrm{KBr}, \mathrm{cm}^{-1}\right): 1597(\mathrm{~m}), 1472(\mathrm{~s}), 1383(\mathrm{~m}), 1230(\mathrm{~m}), 1012(\mathrm{~m})$, $972(\mathrm{w}), 929(\mathrm{~m}), 881(\mathrm{~m}), 817(\mathrm{~m}), 710$ (m), 661 (s). Anal. Calcd for $\mathrm{C}_{36} \mathrm{H}_{70} \mathrm{Cl}_{2} \mathrm{Cu}_{2} \mathrm{P}_{2}: \mathrm{C}, 56.68 ; \mathrm{H}$, 9.25; Cl, 9.29. Found: C, 56.96; H, 9.18; Cl, 8.84.

[[(3-Cyclopropyl-1,2-diphenylpropen-1-yl)diisopropylphosphine $] \mathbf{C u}(\boldsymbol{\mu}-\mathbf{C l})]_{2} \quad$ (5gn). Colorless crystals. mp: $145-146{ }^{\circ} \mathrm{C}(\mathrm{dec}) .{ }^{1} \mathrm{H}$ NMR $\left(\mathrm{CDCl}_{3}\right): \delta$ 0.35-0.42 (m, 8H), 0.64-0.78 (m, 2H), 1.17-1.36 (m, $24 \mathrm{H}), 1.95-2.16(\mathrm{~m}, 4 \mathrm{H}), 3.32(\mathrm{~d}, J=6.7 \mathrm{~Hz}, 4 \mathrm{H}), 6.84-7.12(\mathrm{~m}, 20 \mathrm{H}) .{ }^{13} \mathrm{C} \mathrm{NMR}\left(\mathrm{CDCl}_{3}\right): \delta 5.0,10.4$, 20.0 (d), 20.5 (d), 24.3 (d), 42.0 (d), 126.0, 126.3, 127.2, 127.5, 128.3, 129.2 (d), 130.1, 139.6, 142.7 (d), $160.9(\mathrm{~d}) .{ }^{31} \mathrm{P}$ NMR $\left(\mathrm{CDCl}_{3}\right): \delta$ 12.8. IR $\left(\mathrm{KBr}, \mathrm{cm}^{-1}\right): 1598(\mathrm{~m}), 1587(\mathrm{~m}), 1574(\mathrm{~m}), 1487$ (s), 1441 (s), $1383(\mathrm{~m}), 1246$ (m), 1047 (s), 1014 (s), 765 (s), 700 (s). Anal. Calcd for $\mathrm{C}_{48} \mathrm{H}_{62} \mathrm{Cl}_{2} \mathrm{Cu}_{2} \mathrm{P}_{2}: \mathrm{C}, 64.13 ; \mathrm{H}$, 6.95; Cl, 7.89. Found: C, 63.90; H, 7.09; Cl, 7.62. 

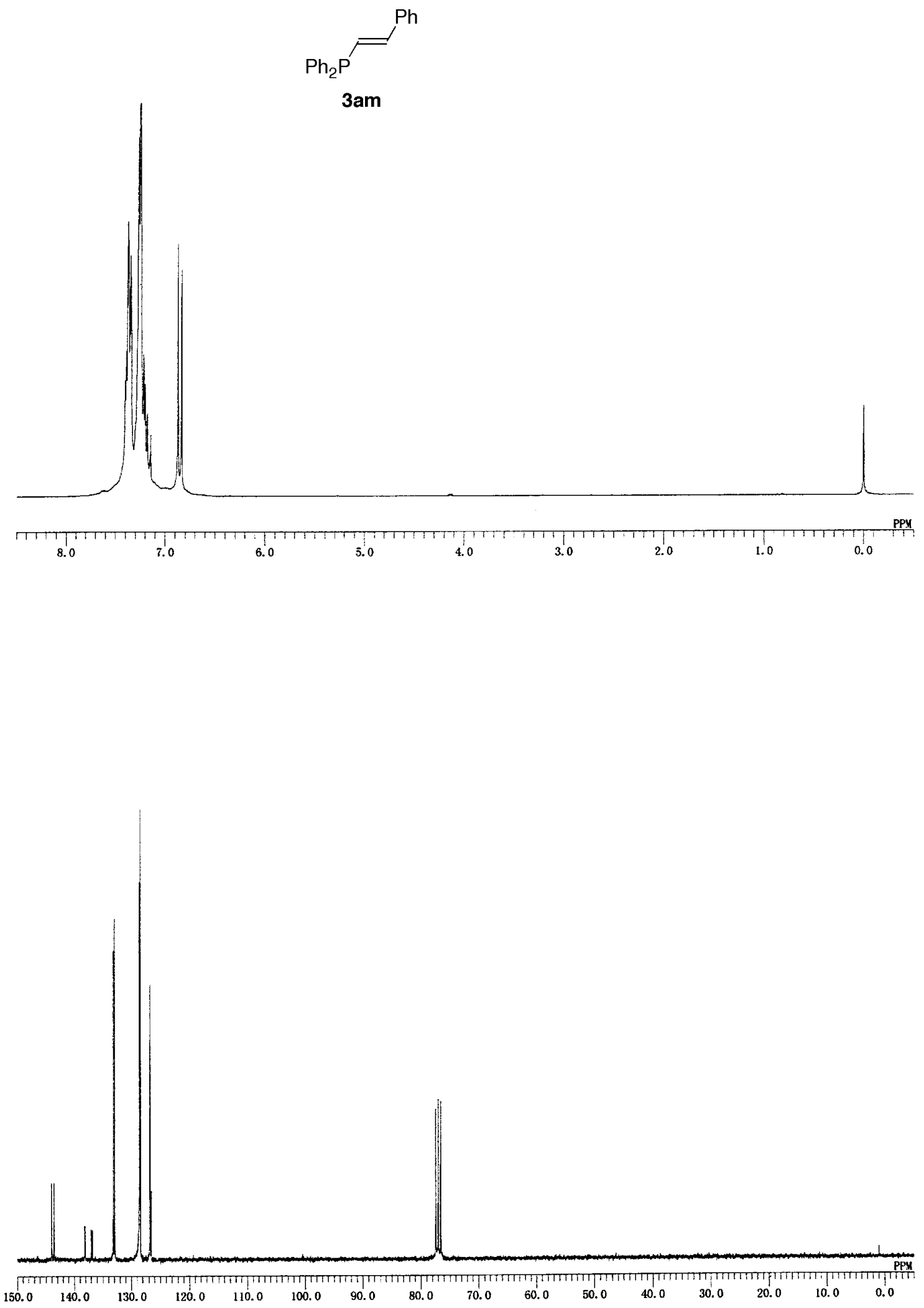

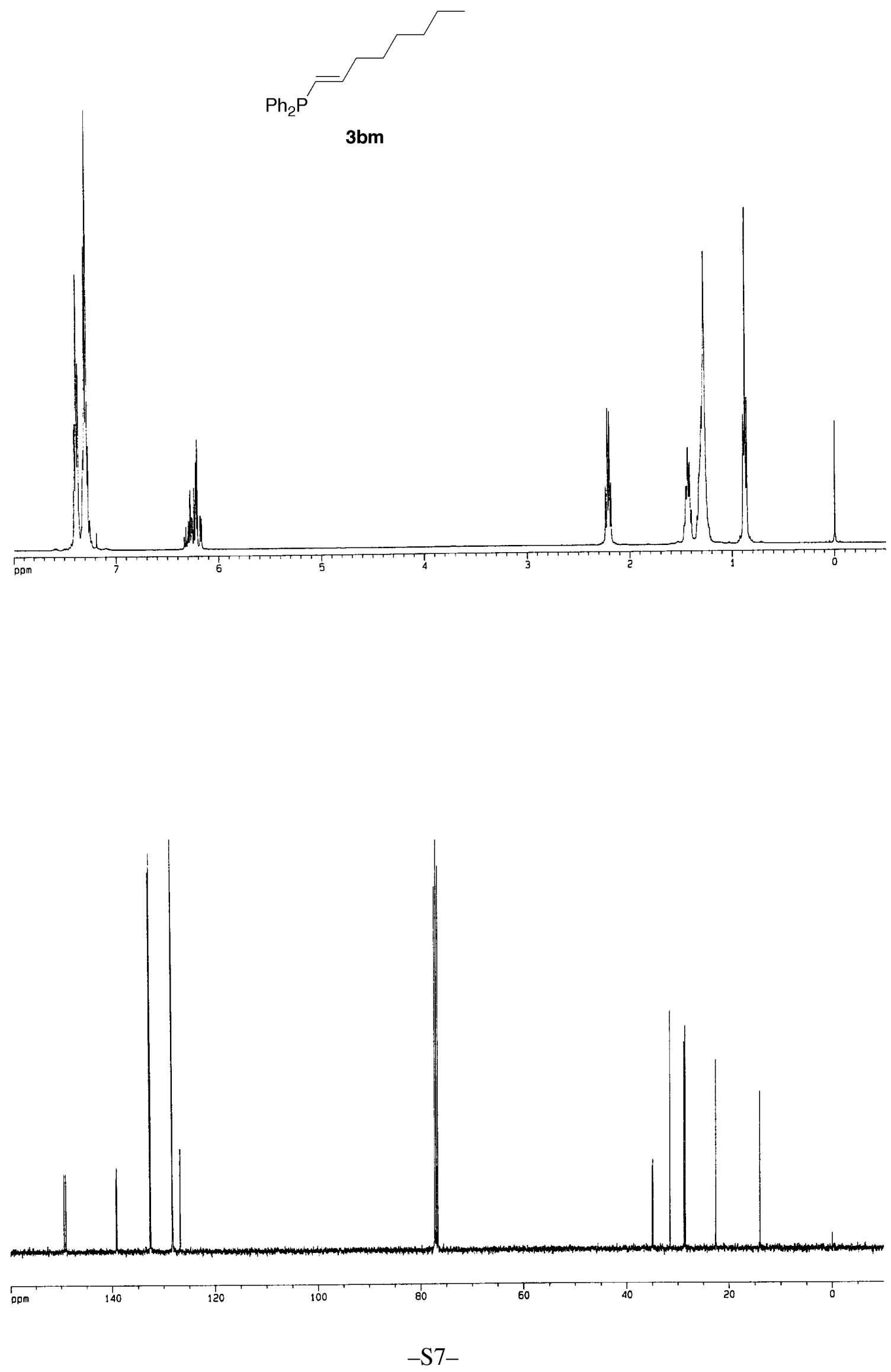

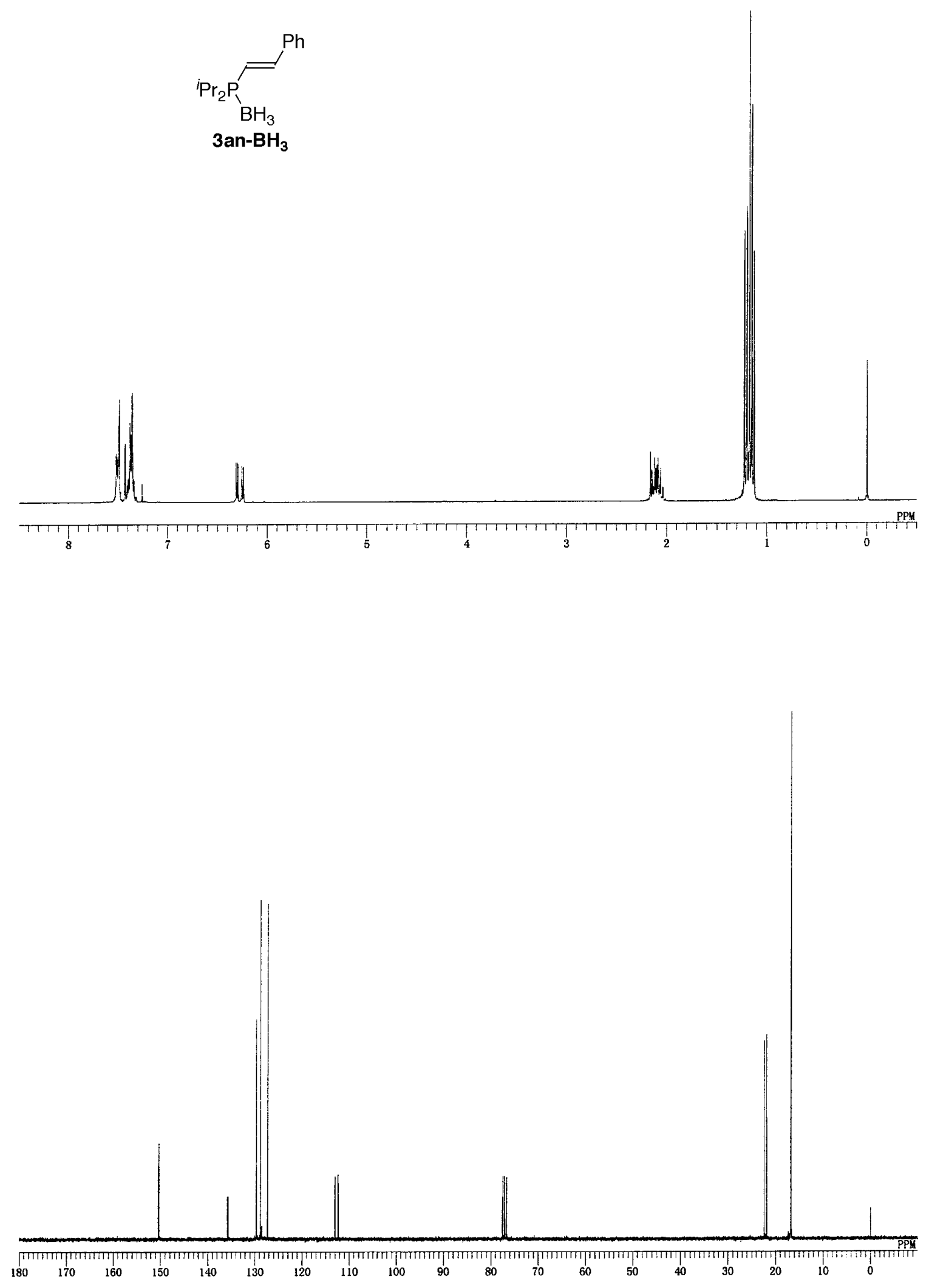

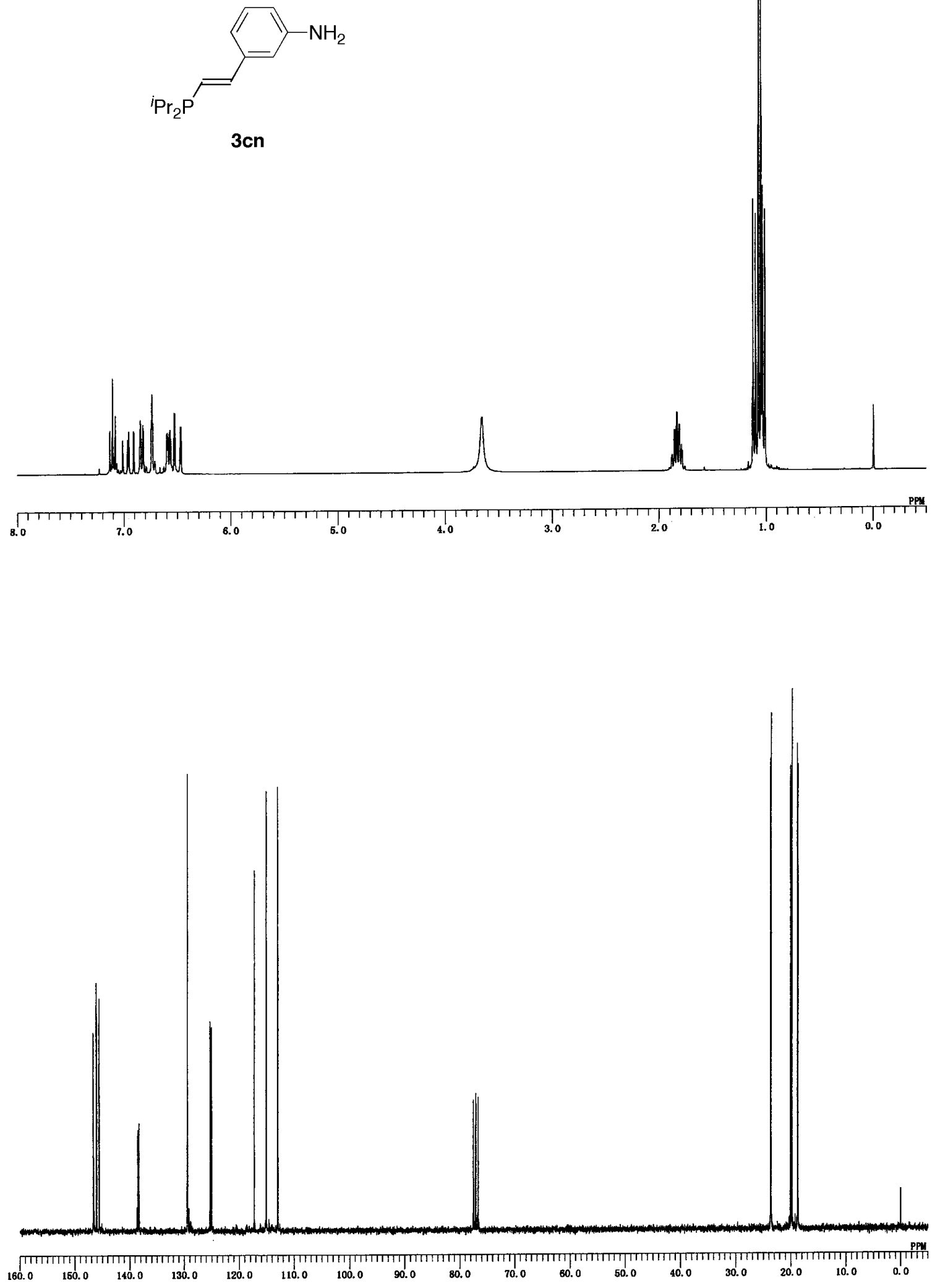

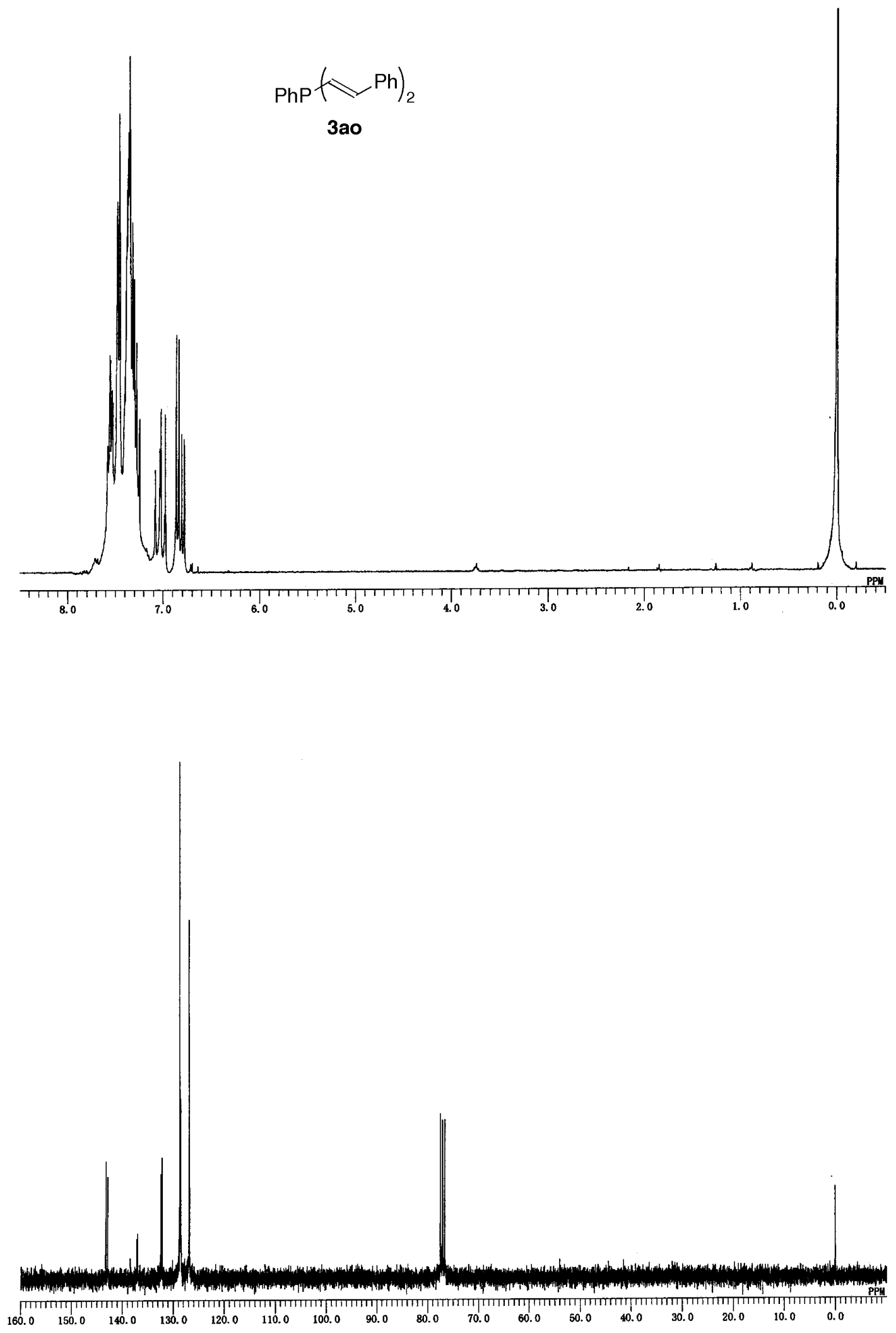

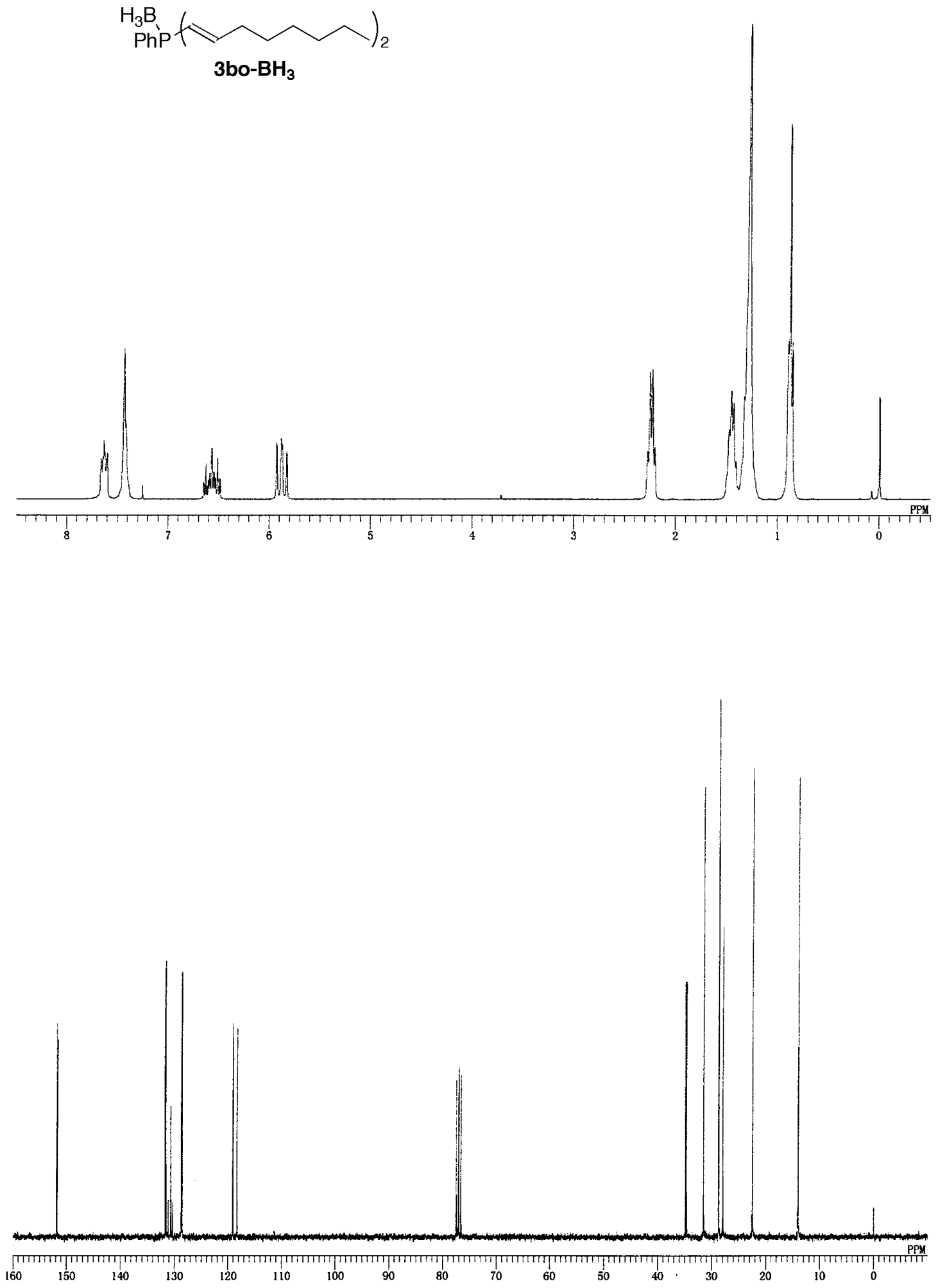

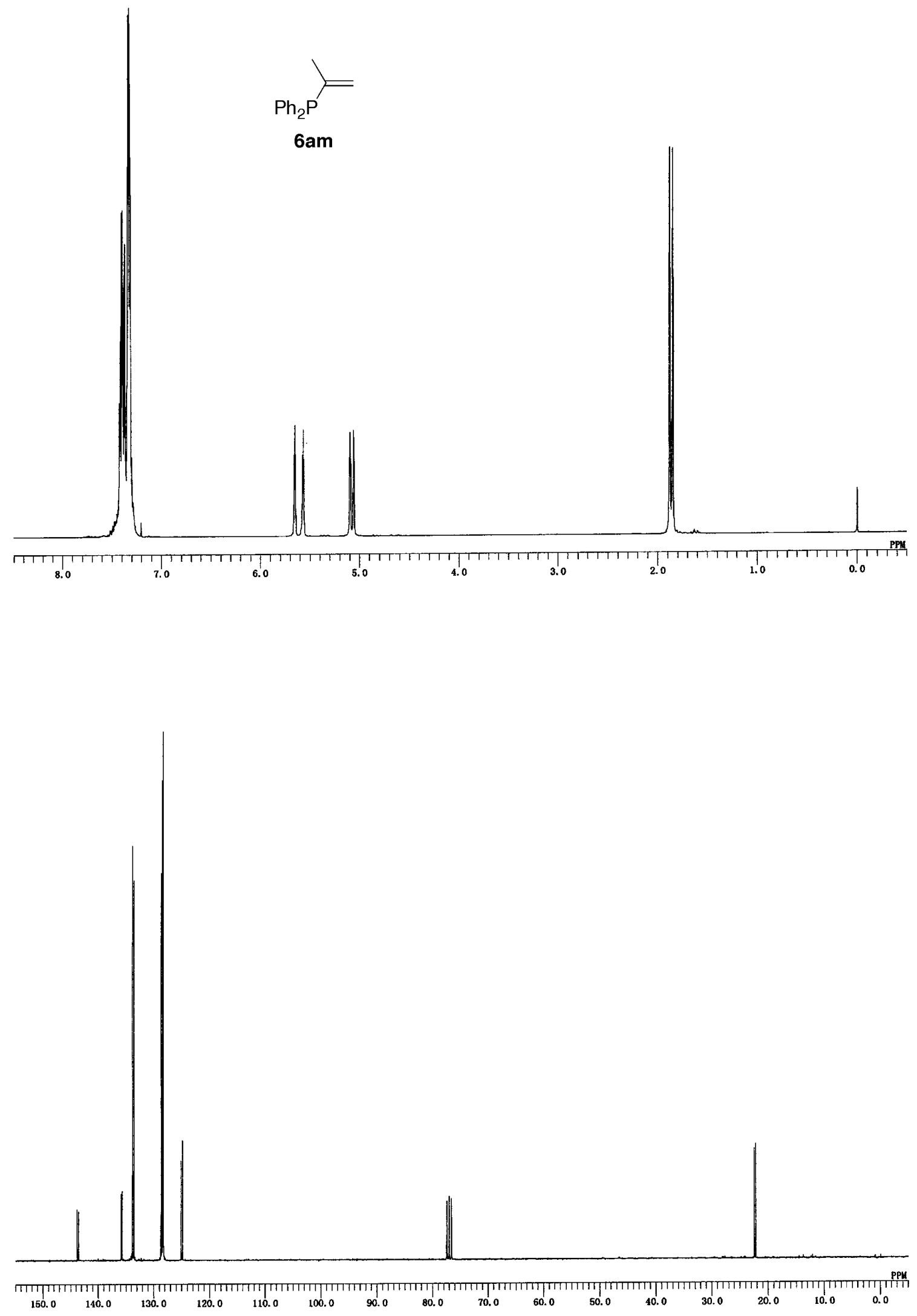

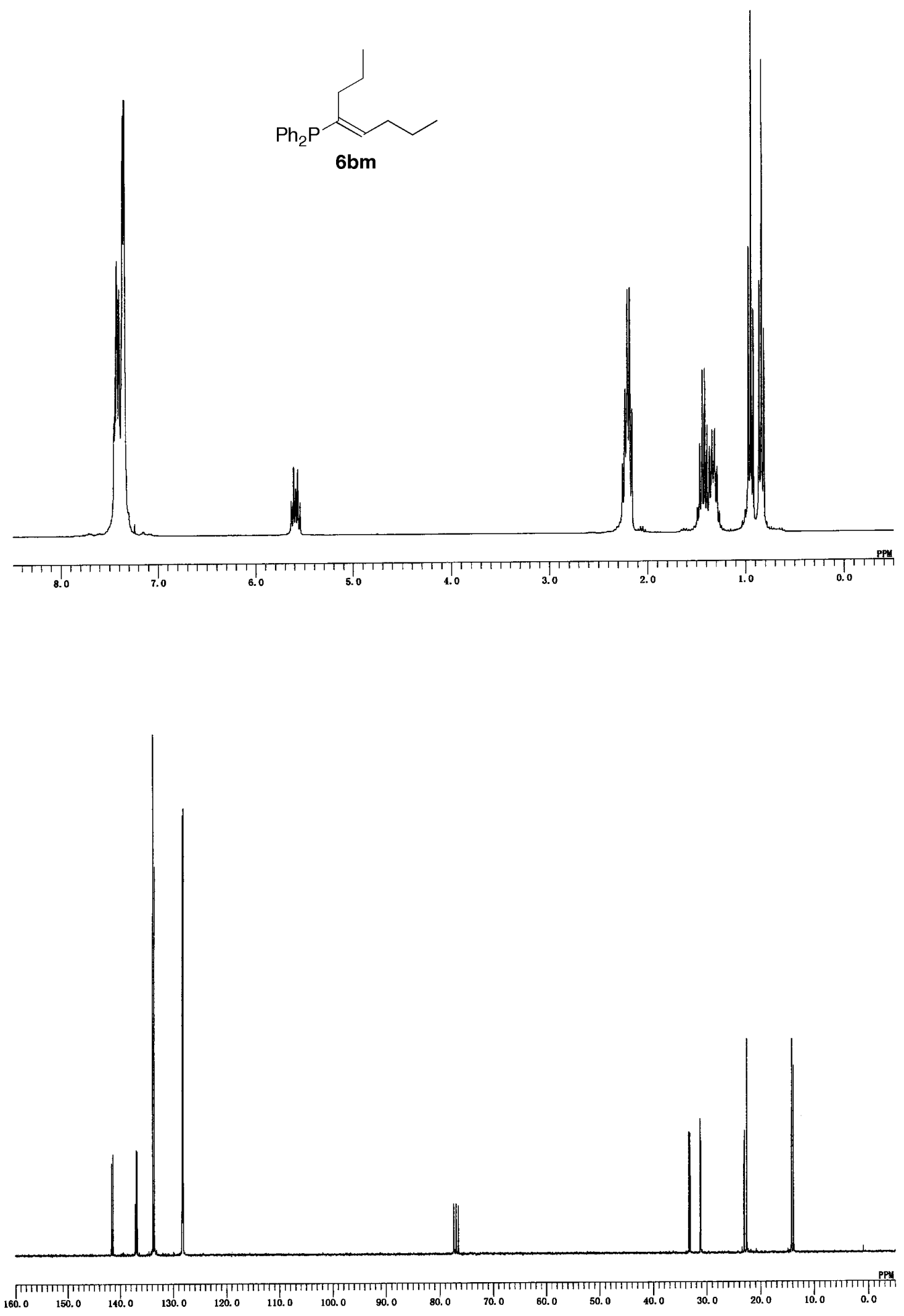

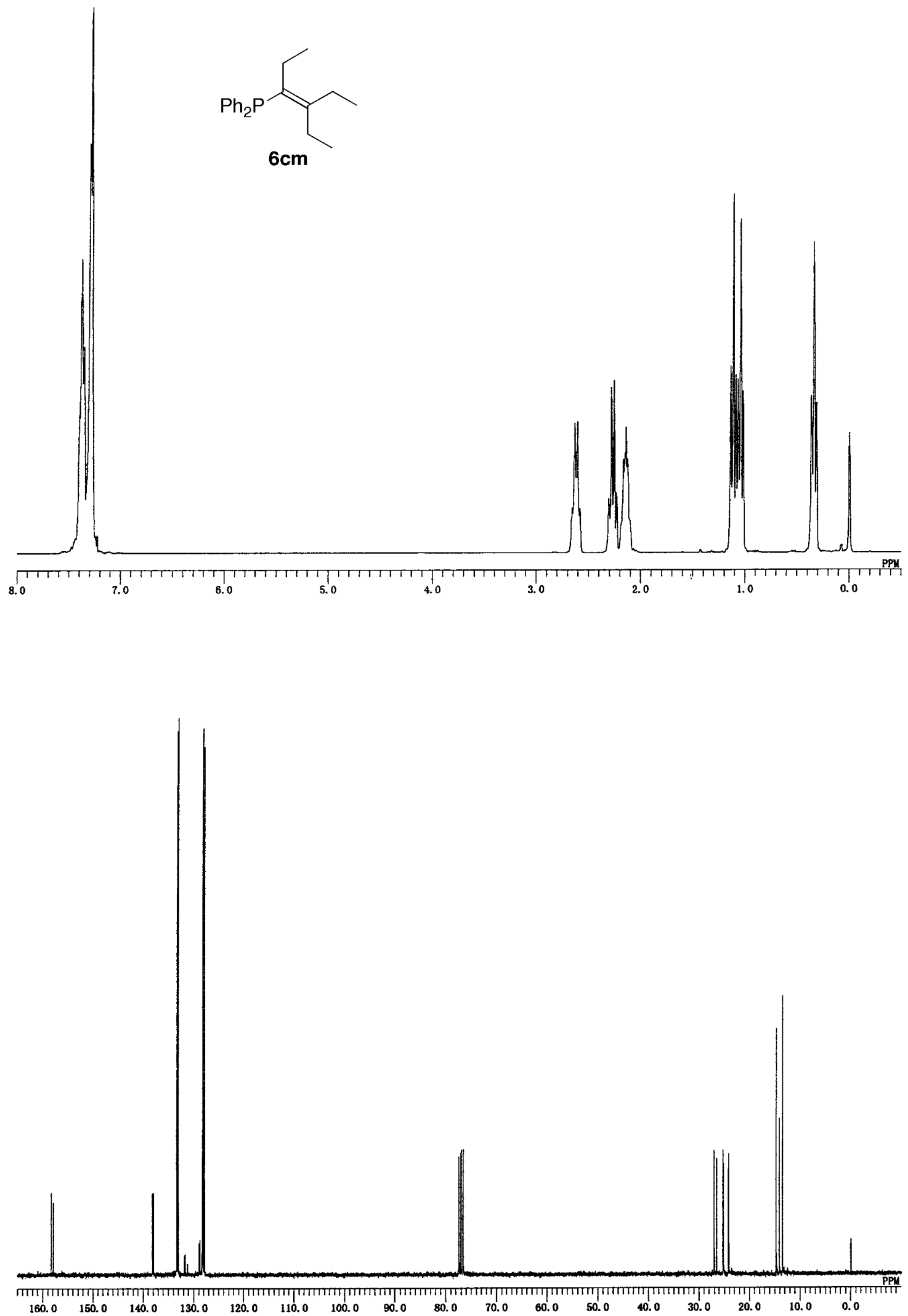

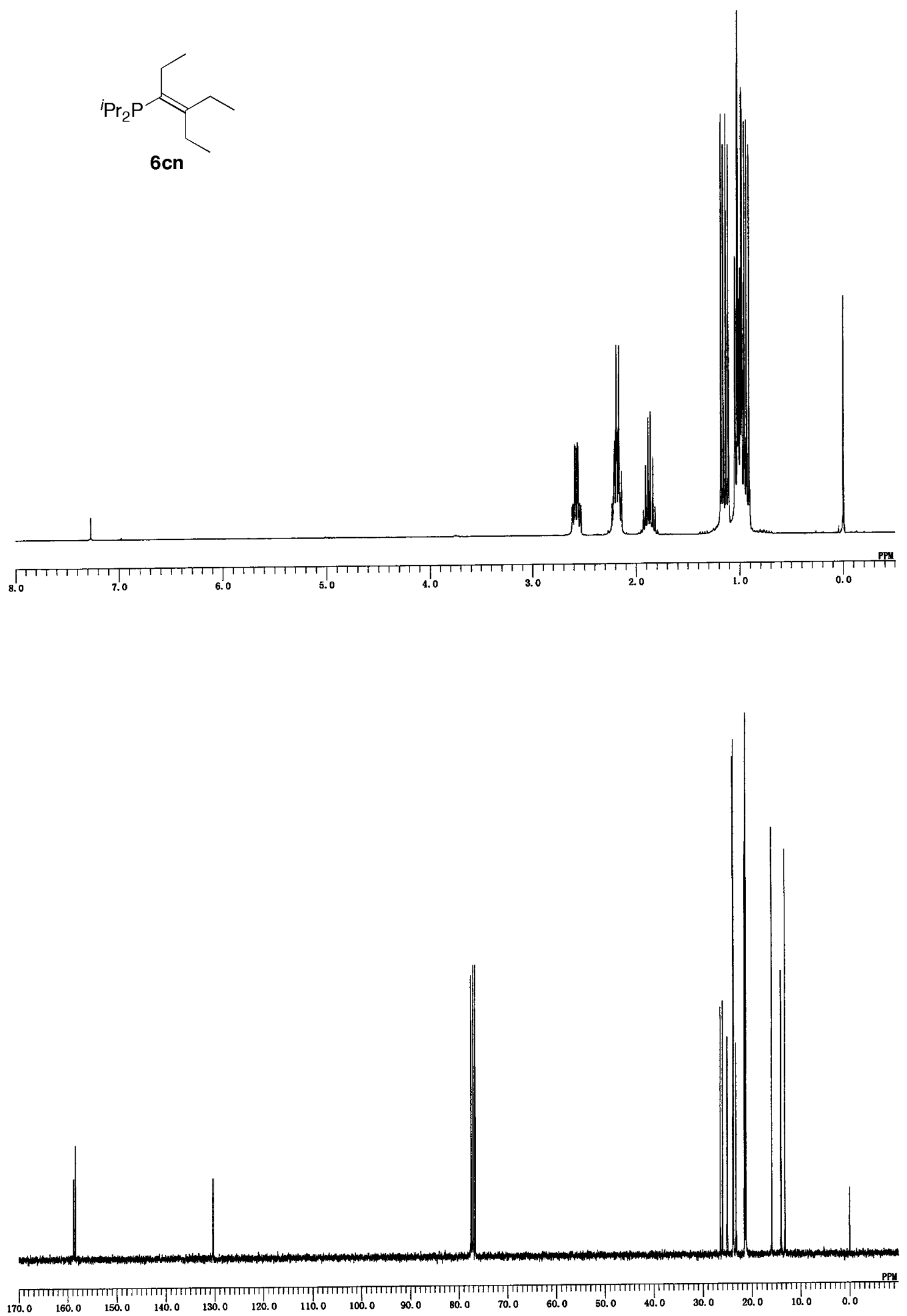

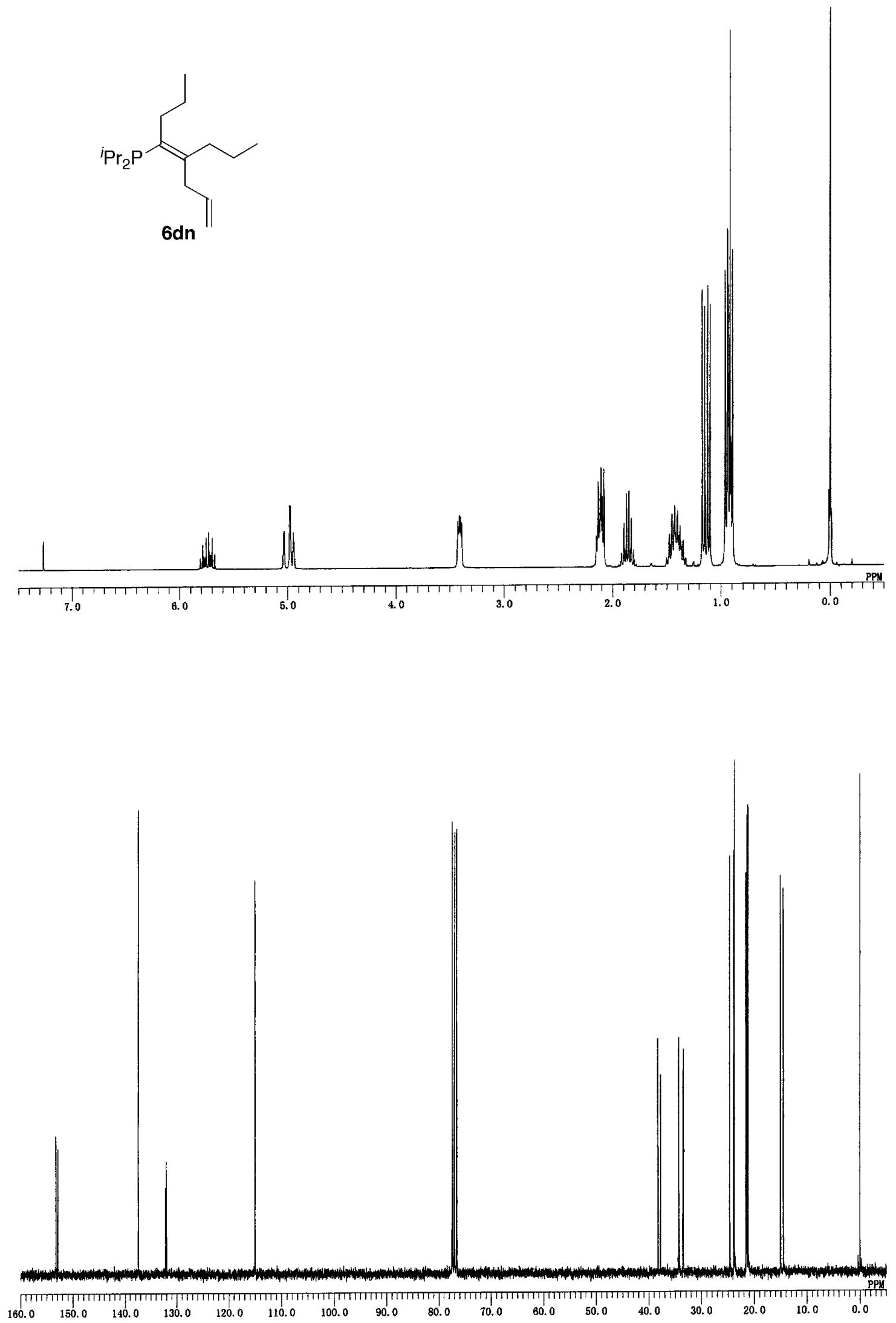

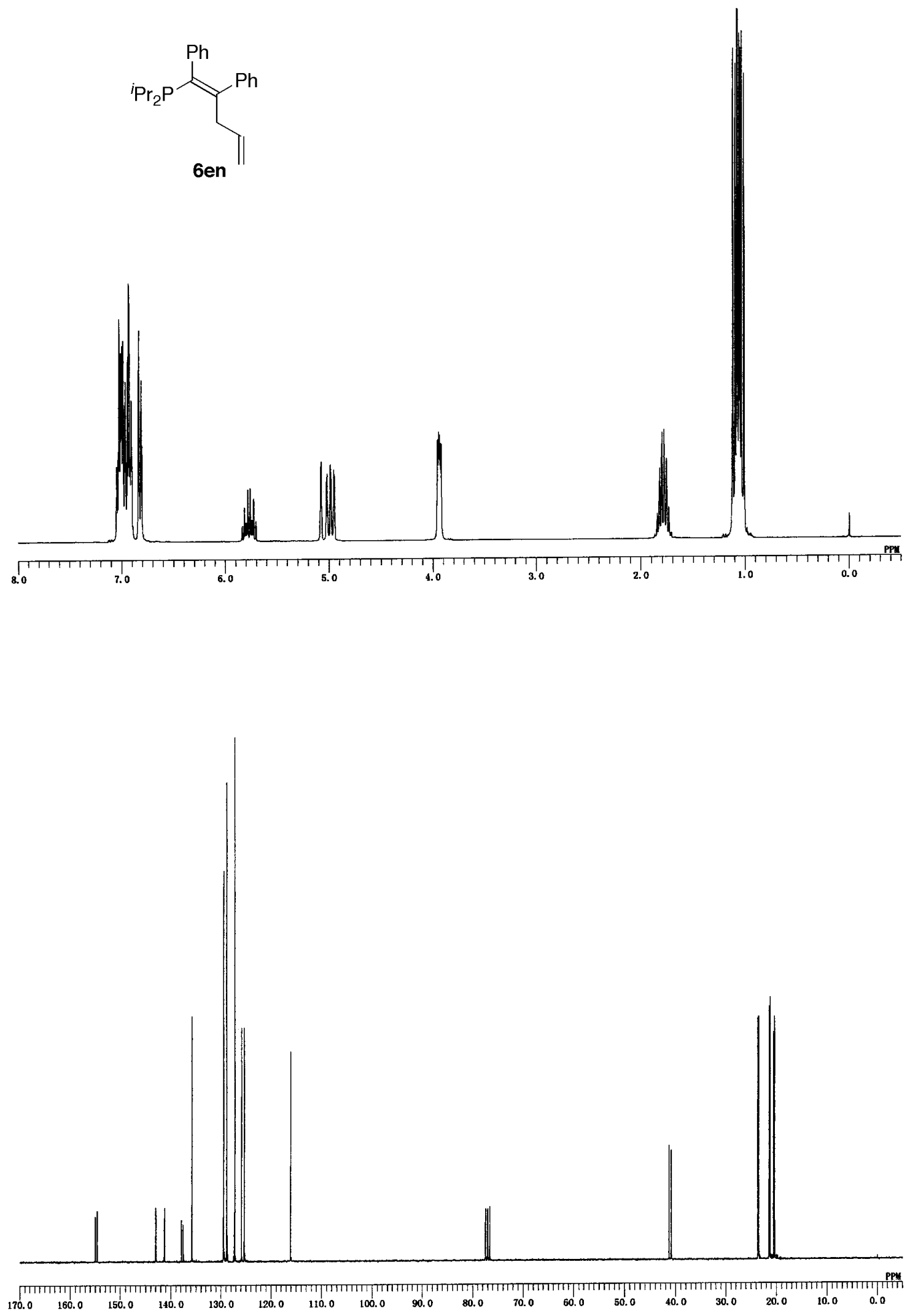

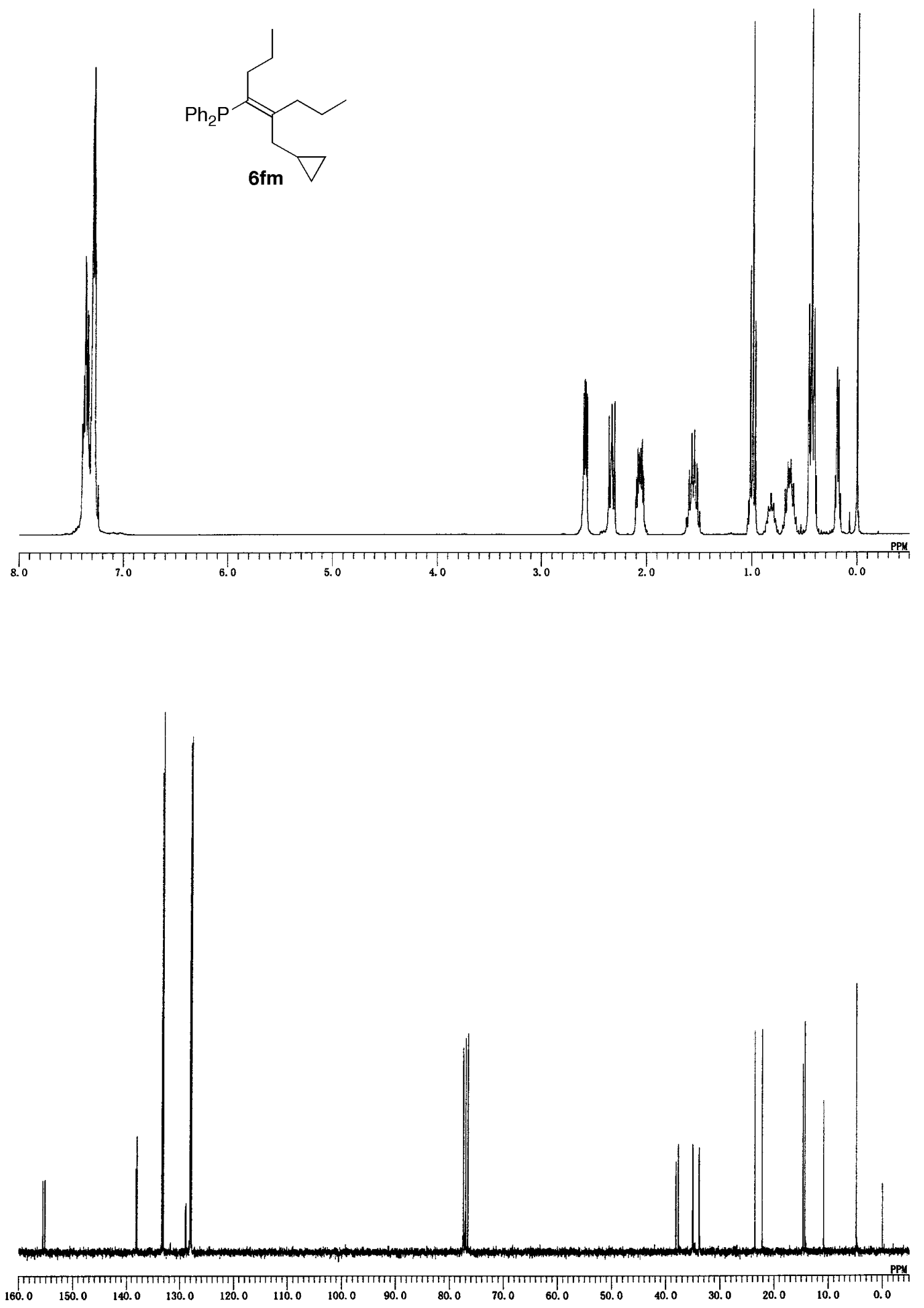

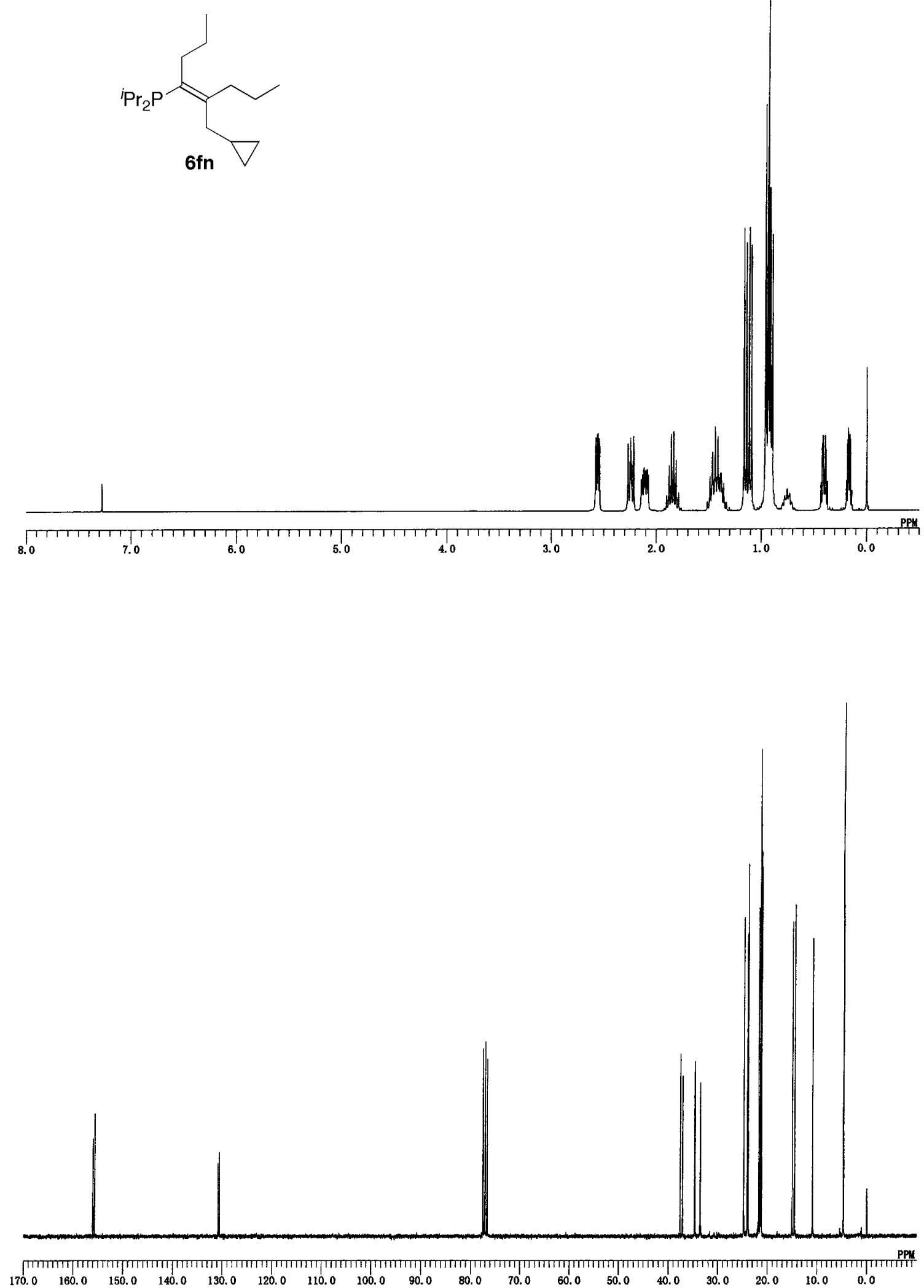

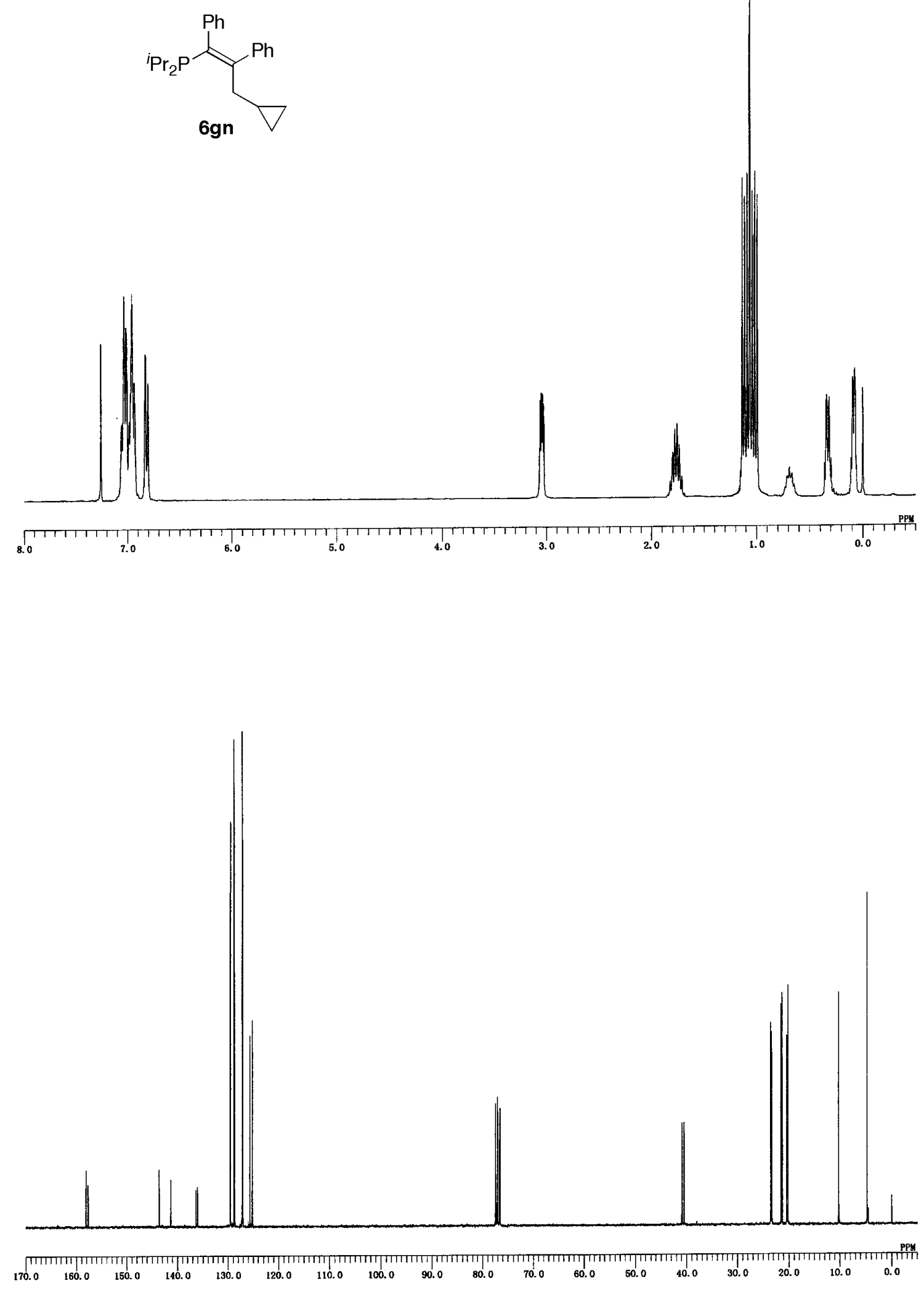

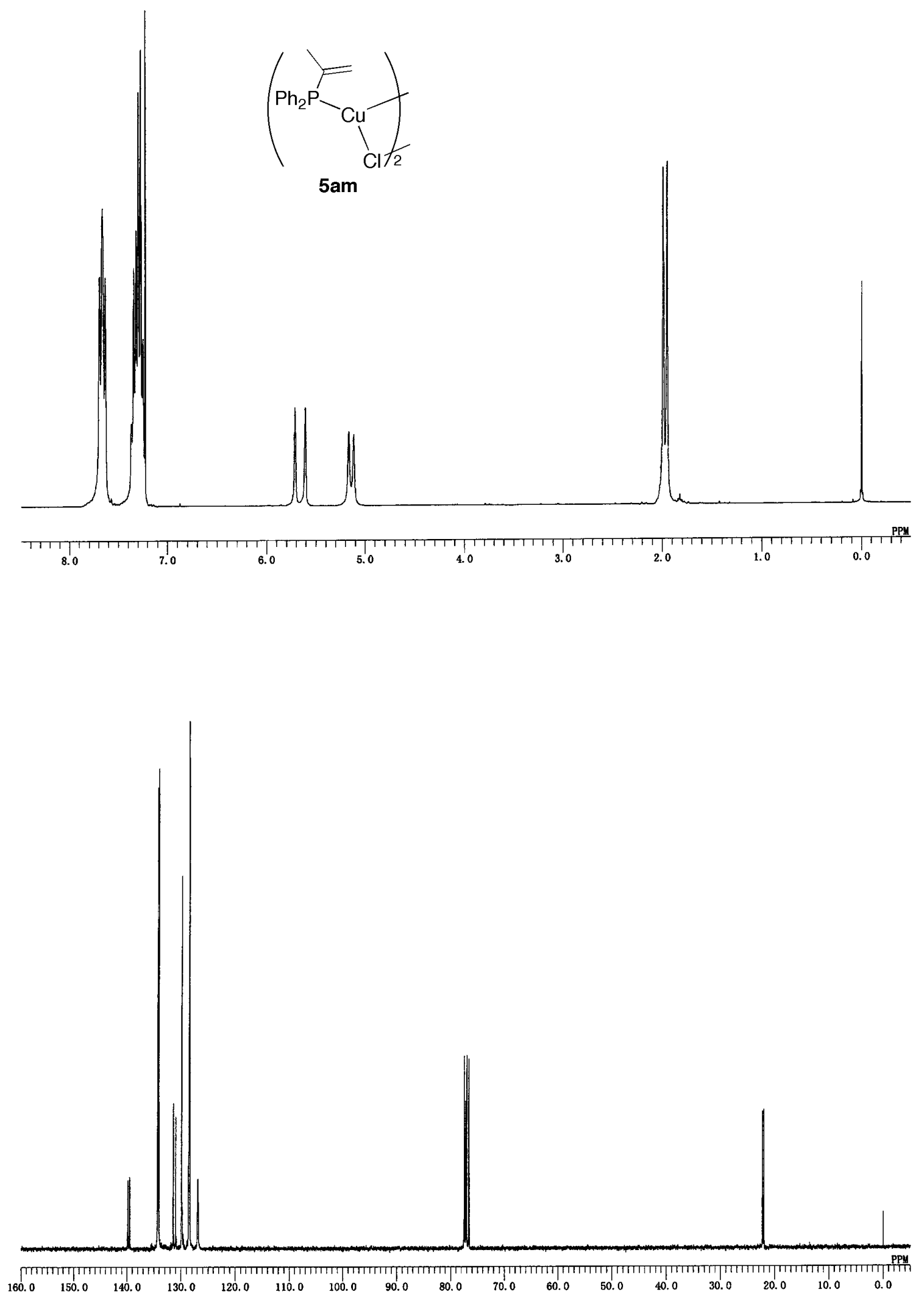

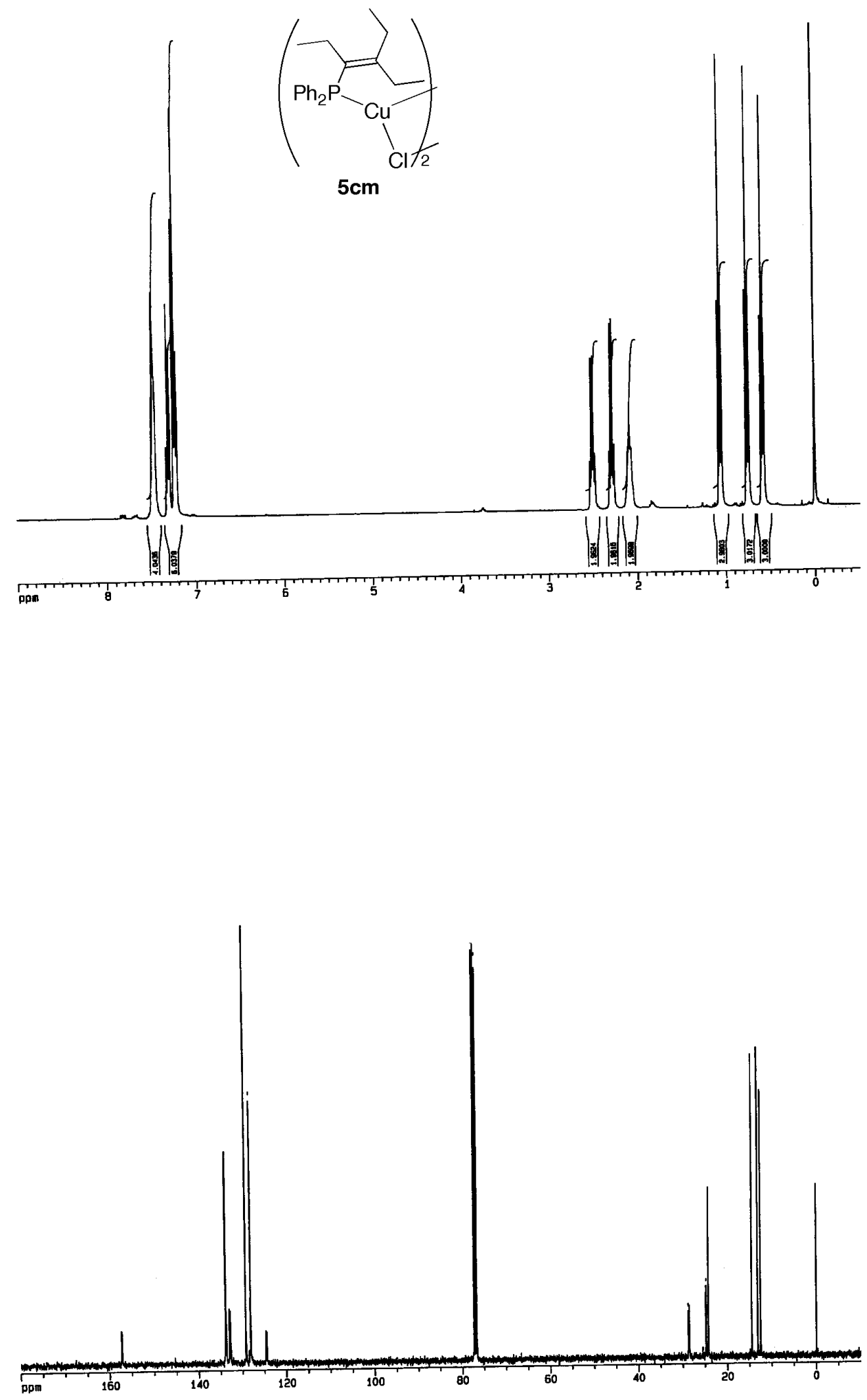

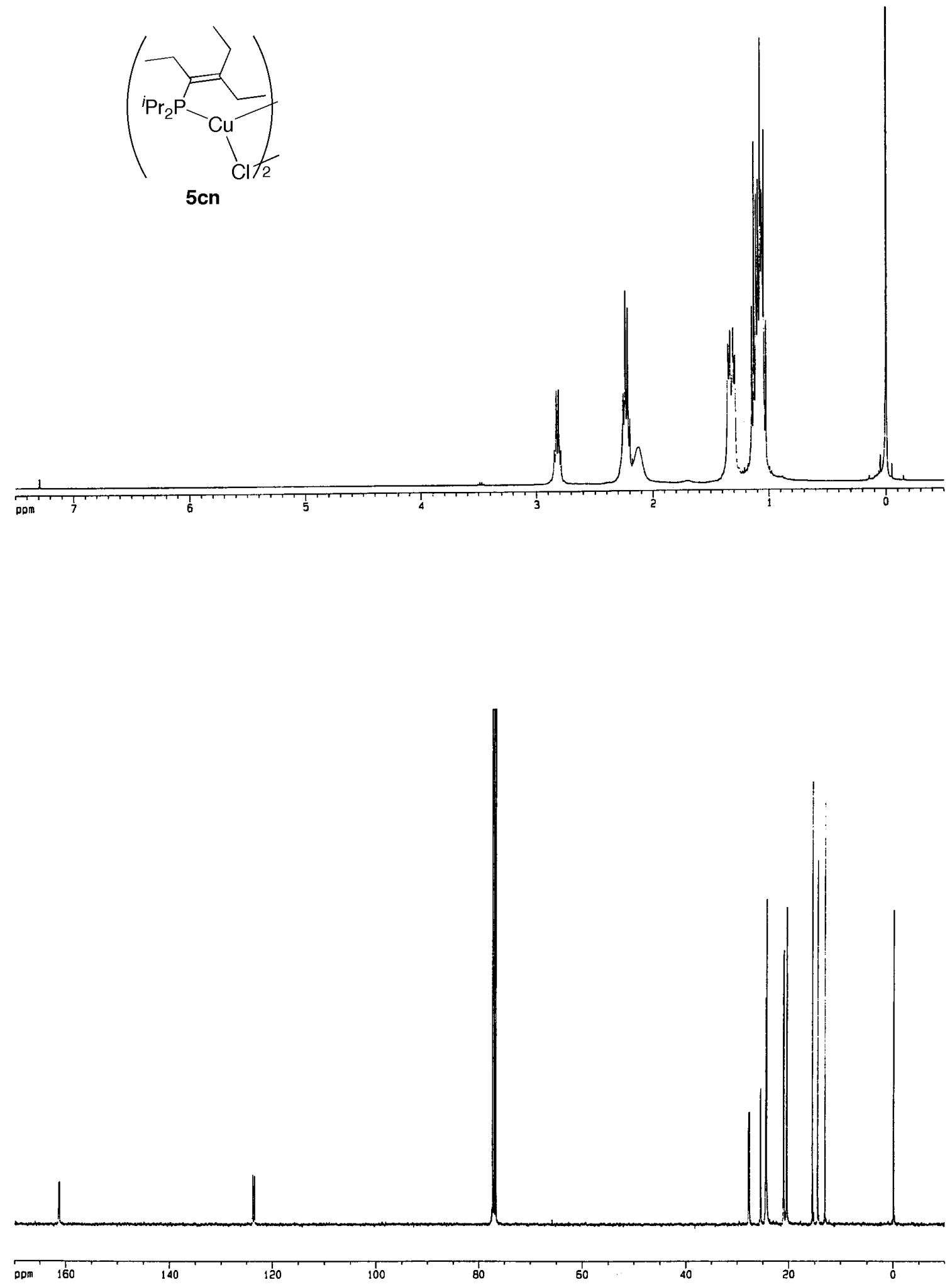

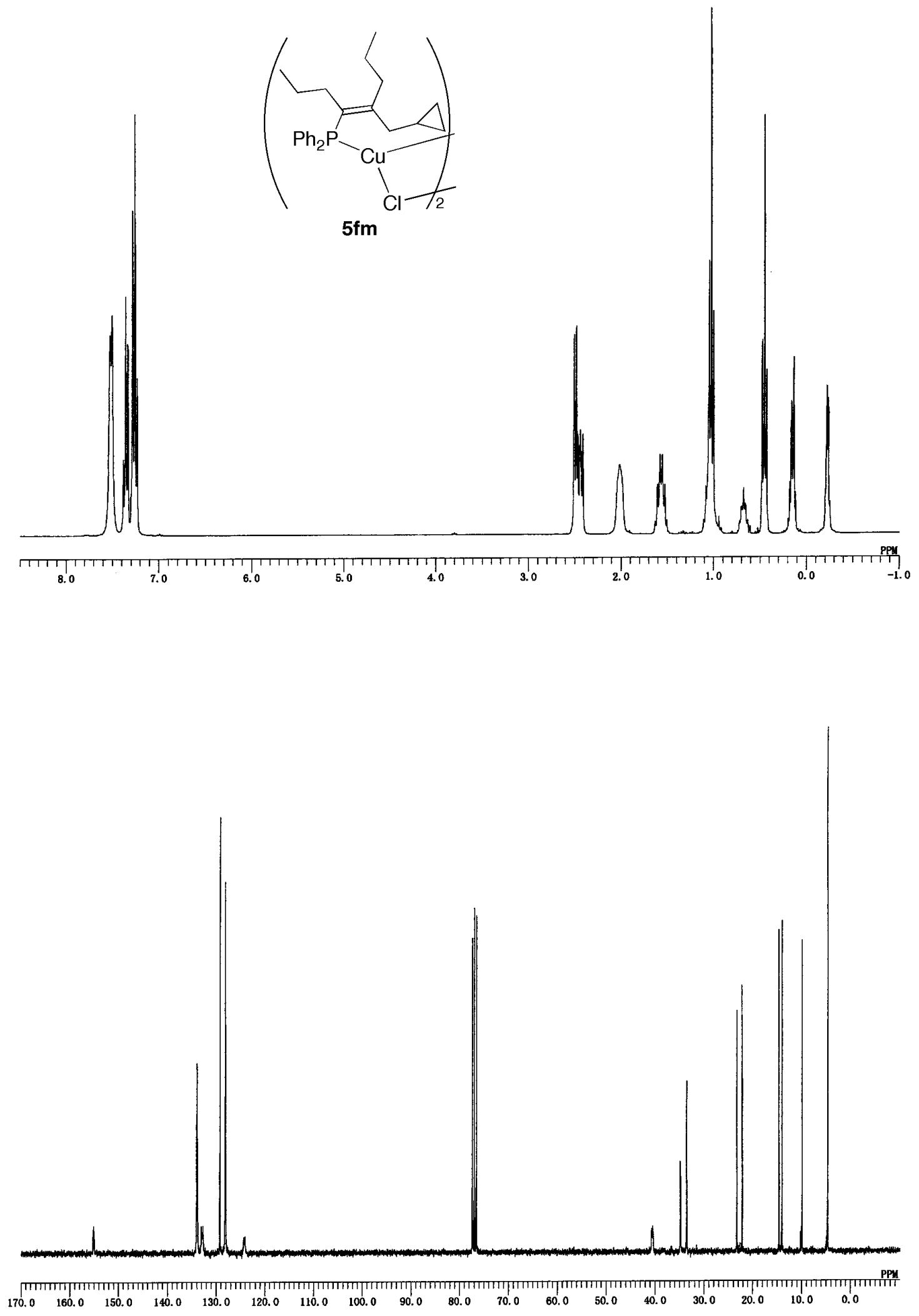

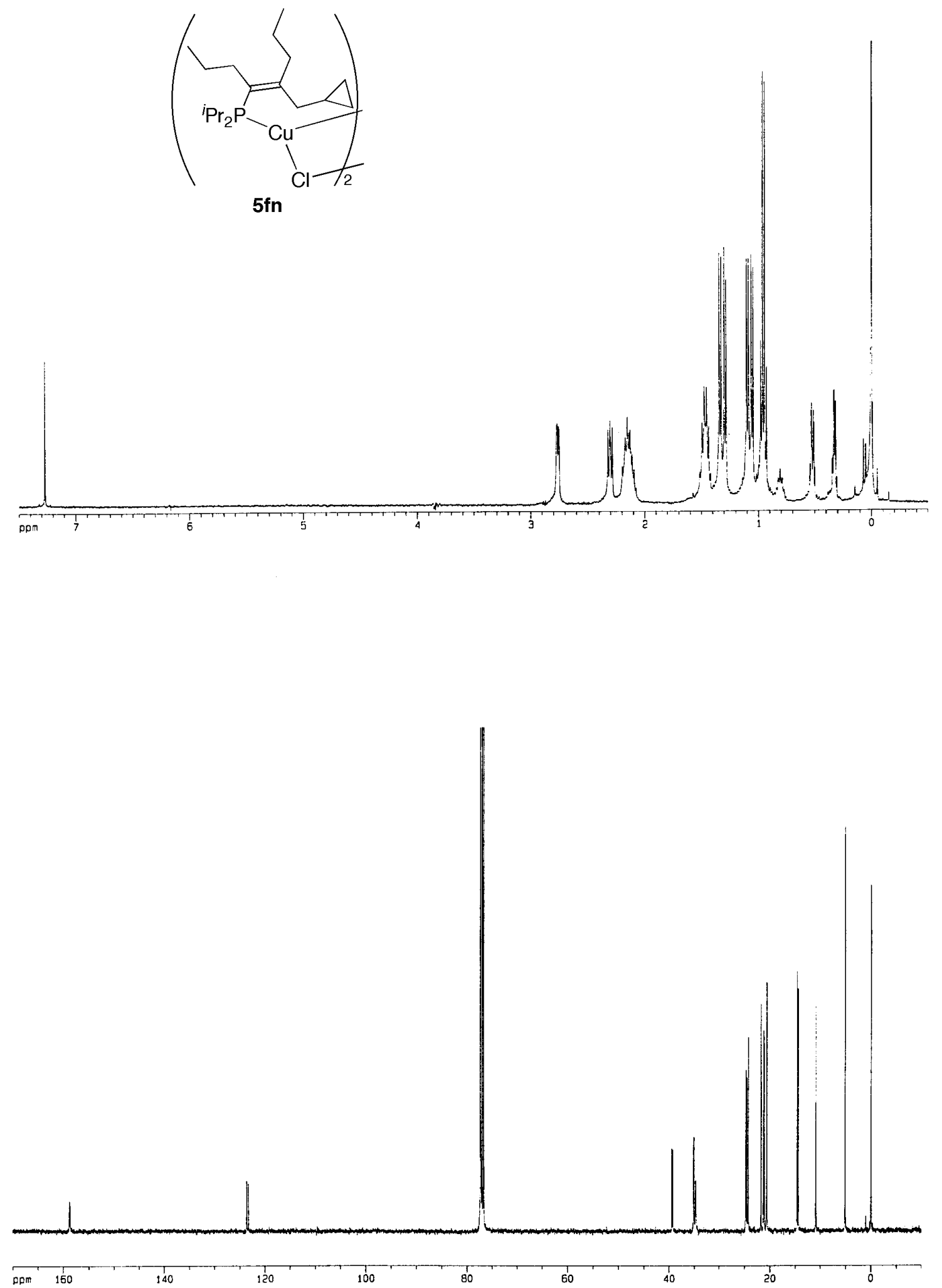

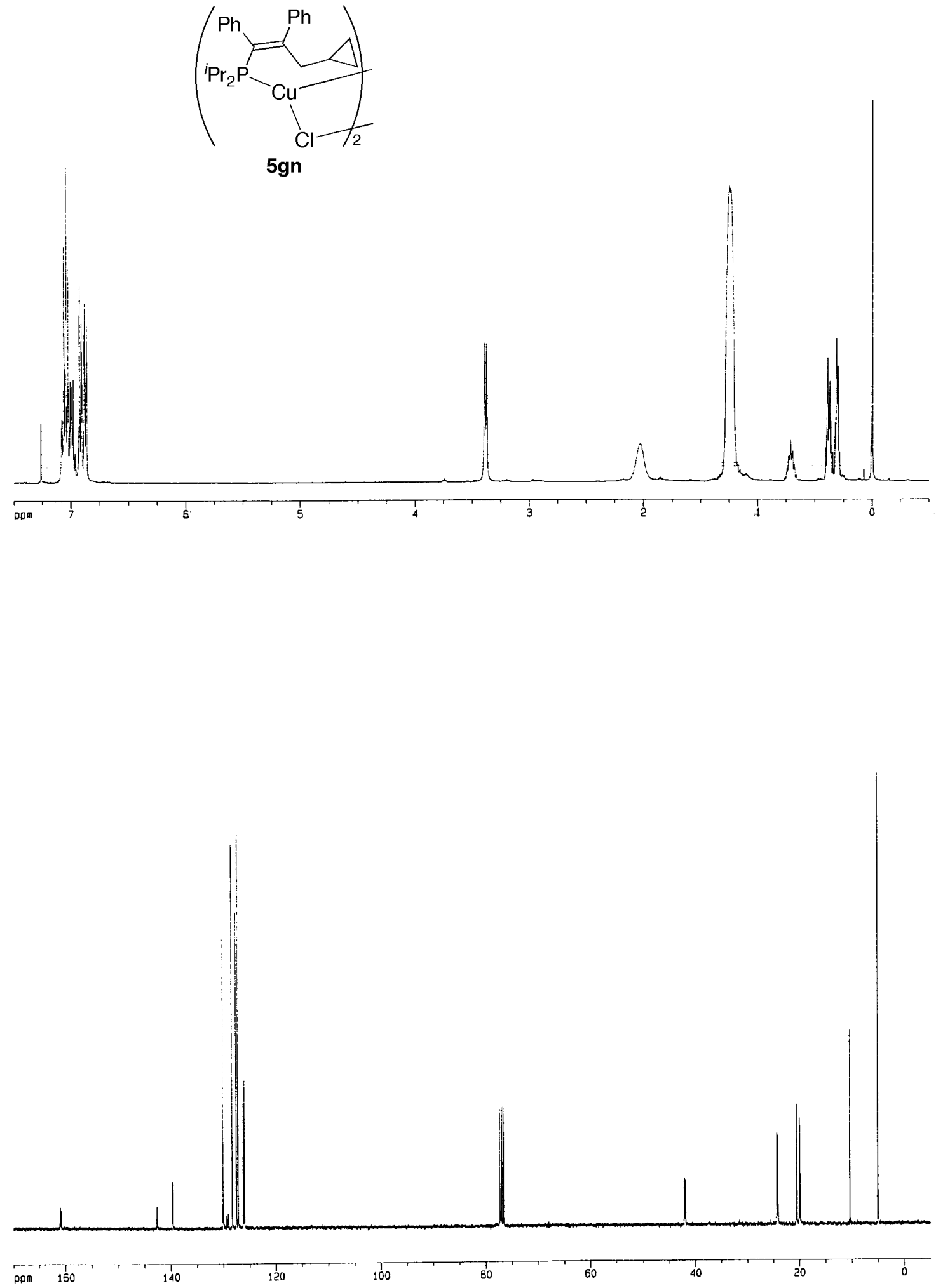